\title{
Parametric dependence of irregular conformal block
}

\author{
Sang-Kwan Choi and Chaiho Rim \\ Department of Physics and Center for Quantum Spacetime, \\ Sogang University, Seoul 121-742 Korea \\ E-mail: hermit1231@gmail.com, rimpine@sogang.ac.kr
}

ABSTRACT: Irregular conformal block is an important tool to study a new type of conformal theories, which can be constructed as the colliding limit of the regular conformal block. The irregular conformal block is realized as the $\beta$-deformed Penner matrix model whose partition function is regarded as the inner product of the irregular modules. The parameter dependence of the inner product is obtained explicitly using the loop equation with close attention to singularities in the parameter space. It is noted that the exact singular structure of the parameter space in general can be found using a very simple and powerful method which uses the flow equations of the partition function together with the hierarchical structure of the singularity. This method gives the exact expression to all orders of large $N$ expansion without using the explicit contour integral of the filling fraction.

KeYwords: Nonperturbative Effects, Matrix Models, Conformal and W Symmetry

ARXiv EPRINT: 1312.5535 


\section{Contents}

1 Introduction 1

2 Irregular conformal block $\quad 4$

2.1 Loop equation and flow equation 4

$\begin{array}{ll}2.2 & \text { Inner product }\left\langle\Delta \mid I^{(m)}\right\rangle\end{array}$

$\begin{array}{lll}2.3 & \text { Inner product }\left\langle I^{(n)} \mid I^{(m)}\right\rangle & 10\end{array}$

3 Hierarchical structure of the partition function $\quad 13$

3.1 Singular structure in $\left\langle I^{(n)} \mid I^{(m)}\right\rangle$ and $\left\langle\Delta \mid I^{(m)}\right\rangle \quad 13$

3.2 Hierarchical relation between $\left\langle\Delta \mid I^{(m)}\right\rangle$ and $\left\langle\Delta \mid I^{(m-1)}\right\rangle \quad 16$

$\begin{array}{lll}3.3 & \text { Flow equations for } T^{(k)}\left(\beta^{(k)} ; N_{k}\right) & 18\end{array}$

$\begin{array}{lll}3.4 & \text { Evaluation of } T^{(k)} \text { from the flow equation } & 19\end{array}$

$\begin{array}{lll}4 & \text { Summary and discussion } & 23\end{array}$

$\begin{array}{ll}\text { A Self-consistency of the flow equation for } \tilde{T}^{(k)} & \mathbf{2 4}\end{array}$

\section{Introduction}

In conformal field theory one constructs the lowest weight representation using the Virasoro lowest weight state $|\Delta\rangle[1]$. Recently, however, so called irregular vector was introduced in [2] in connection with the asymptotically free $\mathcal{N}=2$ gauge theories. The irregular vector $\left|I^{(m)}\right\rangle$ is annihilated by positive Virasoro generators $L_{\ell}\left|I^{(m)}\right\rangle=0$ when $\ell>2 m>0$, but is a simultaneous eigenstate of a set of Virasoro generators

$$
L_{k}\left|I^{(m)}\right\rangle=\Lambda_{k}\left|I^{(m)}\right\rangle, \quad m \leq k \leq 2 m
$$

where $\Lambda_{k}$ is a non-vanishing eigenvalue. The existence of the irregular vector is consistent with the Virasoro algebra $\left[L_{k}, L_{n}\right]=(k-n) L_{k+n}$ with $k, n>0$.

The irregular vector can be constructed as the superposition of lowest state together with its descendents. Explicit construction for small $m$ can be found in [2-4]. A more detailed investigation is done in $[5,6]$ in connection with Argyres-Douglas type gauge theories $[7,8]$. It is also noted in [6] that the irregular vector can be constructed in the process of colliding limit of primary fields. This is because the colliding limit of primary fields induces higher than 2 degree of singularities in the operator product expansion with the energymomentum tensor. The higher singularity is the manifestation of the irregular vector.

Let us construct a multi-point state $\left|R_{m}\right\rangle=\lim _{z_{0} \rightarrow 0} \prod_{r=0}^{m} \Psi_{\Delta_{r}}\left(z_{r}\right)|0\rangle$ where $\Psi_{\Delta_{r}}\left(z_{r}\right)$ is the primary field with conformal dimension $\Delta_{r}$. We put $\Psi_{\Delta_{0}}\left(z_{0}\right)$ at the origin so that the 
lowest weight state $\left|\Delta_{0}\right\rangle=\lim _{z \rightarrow 0} \Psi_{\Delta_{0}}(z)|0\rangle$ is obtained. The operator product expansion with the energy-momentum tensor $T(z)$ has the form

$$
T(z)\left|R_{m}\right\rangle=\sum_{r=0}^{m}\left(\frac{\Delta_{r}}{\left(z-z_{r}\right)^{2}}+\frac{1}{\left(z-z_{r}\right)} \frac{\partial}{\partial z_{r}}+\text { regular terms }\right)\left|R_{m}\right\rangle .
$$

The colliding limit is obtained if $z_{r} \rightarrow 0$ but $\Delta_{r} \rightarrow \infty$ so that there appear non-vanishing parameters;

$$
c_{0}=\sum_{r=0}^{m} \alpha_{r}, \quad c_{k}=\sum_{r=0}^{m} \alpha_{r} \sum_{\substack{0 \leq s_{1}<\cdots<s_{k} \leq m \\ s_{i} \neq r}} \prod_{i=1}^{k}\left(-z_{s_{i}}\right) \quad \text { when } 0 \leq k \leq m
$$

where $\Delta_{r}=\alpha_{r}\left(Q-\alpha_{r}\right)$ is used. Then, higher singularities in the OPE with $T(z)$ are induced at the colliding limit

$$
\begin{aligned}
T(z)\left|I^{(m)}\right\rangle & =\left(\sum_{k=0}^{2 m} \frac{\mathcal{L}_{k}}{z^{k+2}}+\frac{L_{-1}}{z}+\text { reg. }\right)\left|I^{(m)}\right\rangle \\
\left|I^{(m)}\right\rangle & =\lim _{z_{r} \rightarrow 0, \alpha_{r} \rightarrow \infty} \prod_{0 \leq r<s \leq m}\left(z_{r}-z_{s}\right)^{2 \alpha_{r} \alpha_{s}}\left|R_{m}\right\rangle
\end{aligned}
$$

where we compensate the position singularity of $\left|R_{m}\right\rangle$ by applying the products of $\left(z_{r}-z_{s}\right)$ 's so that the irregular vector $\left|I^{(m)}\right\rangle$ is well-defined. The induced operator $\mathcal{L}_{k}$ is the realization of the Virasoro algebra on the irregular vector space.

$$
\mathcal{L}_{k}=\Lambda_{k}+\sum_{\ell \in \mathbb{N}}(\ell-k) c_{\ell} \frac{\partial}{\partial c_{\ell-k}}, \quad \Lambda_{k}=(k+1) Q c_{k}-\sum_{\ell=0}^{k} c_{\ell} c_{k-\ell},
$$

where the notation is used: $c_{\ell} \equiv 0$ unless $0 \leq k \leq m$. It is obvious that $\mathcal{L}_{k}=\Lambda_{k}$ for $m \leq k \leq 2 m$ and is consistent with (1.1).

Our task is to investigate the parametric dependence of the irregular vector. Instead of constructing directly as in $[2-4,6]$, we will use the Penner-type matrix model. The Penner model was first introduced to find the Euler characteristic of moduli space of Riemann surfaces with genus and punctures [9]. Soon after, the Penner model turns out to be very useful to understand $c=1$ string theory $[10,11]$ and is further generalized [12] to obtain the conformal block of the Liouville theory inspired by AGT relation [13]. Note that $(m+2)$ correlation of vertex operators $\left\langle\prod_{r=0}^{m+1} e^{2 \alpha_{r} \phi\left(z_{r}\right)}\right\rangle$ with the Liouville momentum $\alpha_{a}$ is evaluated perturbatively by expanding the Liouville potential $e^{2 b \phi}$, $\left\langle\left(\prod_{i=1}^{N} \int d \lambda_{i} d \bar{\lambda}_{i} e^{2 b \phi\left(\lambda_{i}\right)}\right) \prod_{r=0}^{m+1} e^{2 \alpha_{r} \phi\left(z_{r}\right)}\right\rangle_{0}$ using the free correlation $\left\langle e^{2 \alpha_{1} \phi(z)} e^{2 \alpha_{2} \phi(w)}\right\rangle_{0}=$ $|z-w|^{-4 \alpha_{1} \alpha_{2}}$ with the neutrality condition $\sum_{a=0}^{m+1} \alpha_{a}+b N=Q$. Here, $Q(=b+1 / b)$ is the Liouville background charge. Then, the conformal block $\mathcal{F}_{m+2}\left(\left\{e^{\alpha_{k} \phi\left(z_{k}\right)}\right\}\right)$ (figure 1$)$ is identified with $Z_{N} \times \prod_{0 \leq a<b \leq m+1}\left(z_{a}-z_{b}\right)^{-2 \alpha_{a} \alpha_{b}}$ which defines the $\beta$-deformed Penner-type matrix model:

$$
Z_{N}=\int \prod_{i=1}^{N} d \lambda_{i} \Delta(\lambda)^{2 \beta} e^{-\frac{\sqrt{\beta}}{g} \sum_{i} V\left(\lambda_{i}\right)}
$$




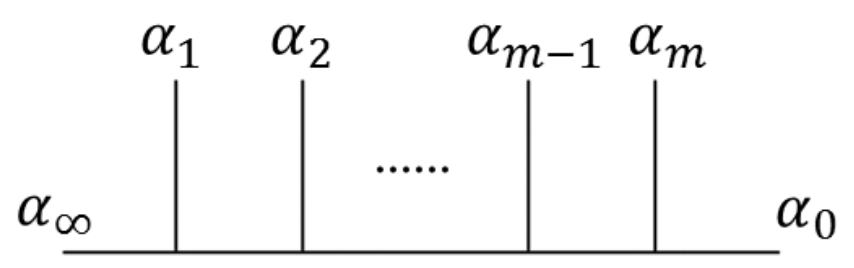

Figure 1. Diagram of a $(m+2)$-point conformal block

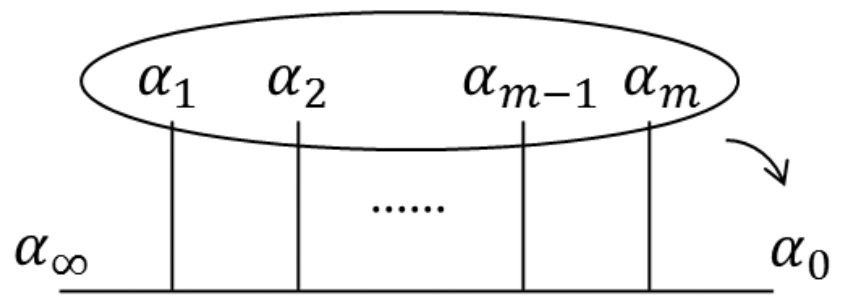

Figure 2. Schematic diagram of $\left\langle\Delta \mid I^{(m)}\right\rangle$. The irregular singularity with rank $m$ is developed when $m$-intermediate points are taken to one point at origin in a correlated way.

where $\Delta(\lambda)=\prod_{i<j}\left(\lambda_{i}-\lambda_{j}\right)$ is the Vandermonde determinant. The potential is given as the sum of logarithmic terms: $V(z)=-\sum_{a=0}^{m} \alpha_{a} \log \left(z-z_{a}\right)$. To make large $N$ expansion possible, we rescale $\alpha_{a} \rightarrow \alpha_{a} / \hbar$ and rename $\beta \equiv-b^{2}$ and $\hbar \equiv-2 i g$ assuming $\hbar=\mathcal{O}(1 / N)$ and $\alpha_{a}=\mathcal{O}(1)$. This $\beta$-deformed Penner type matrix is proved very useful to reproduce the Nekrasov partition function $[14,15]$.

If we put $z_{m+1}=\infty$, we may view the conformal block $\mathcal{F}_{m+2}\left(\left\{e^{\alpha_{k} \phi\left(z_{k}\right)}\right\}\right)$ as the inner product $\left\langle\Delta_{\infty} \mid R_{m}\right\rangle$. The out-state $\left\langle\Delta_{\infty}\right|$ has the Liouville momentum $\alpha_{\infty}$ and is the hermitian conjugation of the in-state $\left|\Delta_{0}\right\rangle$ with $\alpha_{0}$. At the colliding limit (figure 2), $\left|R_{m}\right\rangle$ becomes the irregular vector. With the compensating product of $\left(z_{a}-z_{b}\right)$ 's in $\mathcal{F}_{m+2}\left(\left\{e^{\alpha_{k} \phi\left(z_{k}\right)}\right\}\right)$, $Z_{N}$ becomes $\left\langle\Delta \mid I^{(m)}\right\rangle$ and the potential is given as the sum of logarithmic and inverse power terms

$$
V(z)=-c_{0} \log z+\sum_{k=1}^{m} \frac{c_{k}}{k z^{k}}
$$

We will use the notation $Z_{N}^{(m)}$ for the partition function with this potential (1.8) to distinguish from the one (1.7) which has logarithmic potentials only.

Suppose one changes the integral variables $\lambda_{i} \rightarrow 1 / \lambda_{i}$ in the partition function $Z_{N}^{(m)}$. Under the change of variable, the measure changes as $\prod_{i=1}^{N} d \lambda_{i} \rightarrow \prod_{i=1}^{N} d \lambda_{i} / \lambda_{i}^{2}$ and the Vandermonde determinant as $\Delta(\lambda) \rightarrow \Delta(\lambda) \prod_{k=1}^{N} \lambda_{k}^{(1-N)}$. Exponentiating these extra factors into the potential term and using the neutrality condition one has the partition function with a slightly different potential

$$
V(z)=-\alpha_{\infty} \log z+\sum_{k=1}^{m} \frac{c_{k} z^{k}}{k} .
$$


We may interpret the resulting partition function as the hermitian conjugation satisfying the relation $\left\langle\Delta\left(\alpha_{\infty}\right) \mid I^{(m)}\left(\left\{c_{k}\right\}\right)\right\rangle=\left\langle I^{(m)}\left(\left\{c_{k}\right\}\right) \mid \Delta\left(\alpha_{\infty}\right)\right\rangle[16]$.

This paper is organized as follows. In section 2 we evaluate the Penner-type partition function at the colliding limit. The evaluation is done using the loop equation and the flow equations which was successively used in [16]. Here the filling fraction is used to replace all the unknown quantities. In this way one finds the inner product between irregular vectors as well as the product between regular and irregular vectors.

In section 3, we present a simpler way to find the singular structure of the partition function, the singularity in the sense of the parameter space. This is achieved using the fact that the inner product can be put in a hierarchical form. The unknowns in the flow equations are written in terms of power series of the properly defined parameters. The self-consistency condition for the flow equations provide a certain set of recursion relations. Its initial data is trivially given from the loop equation. The merit of this approach is that one does not have to evaluate the very complicated contour integration at all to connect the filling fraction with the unknowns.

Section 4 is the summary and discussion. In appendix A, the details of the proof are found how the self-consistency of the flow equation determines the singular structure of the inner product.

\section{Irregular conformal block}

In this section, we provide explicit results of $Z_{N}^{(m)}$ for the case $m=1,2,3$. Similar calculation for $m=1,2$ can be found in [16]. Explicit expressions of the partition function in terms of the parameters of the potential are useful to find out the role of parameters in the irregular conformal block. The results will be used to check the calculation done in the next section using a quite different method.

\subsection{Loop equation and flow equation}

We briefly review the method of finding the partition function used in [16]. For simplicity we use the large $N$ limit of the loop equation [17-19]

$$
4 W(z)^{2}-4 V^{\prime}(z) W(z)=f(z)
$$

where $W(z)=(\hbar b / 2)\left\langle\sum_{i} 1 /\left(z-\lambda_{i}\right)\right\rangle$ is the resolvent and $\langle\cdots\rangle$ refers to the expectation value with respect to $Z_{N}^{(m)} . f(z)=2 \hbar b\left\langle\sum_{i}\left(-V^{\prime}(z)+V^{\prime}\left(\lambda_{i}\right)\right) /\left(z-\lambda_{i}\right)\right\rangle \mathrm{i}$ is the quantum correction and is related with the partition function

$$
f(z)=\sum_{k=0}^{m-1} \frac{v_{k}\left(-\hbar^{2} \log Z_{N}^{(m)}\right)}{z^{2+k}}, \quad v_{k} \equiv \sum_{s=1}^{m} s c_{s+k} \frac{\partial}{\partial c_{s}}
$$

where the potential (1.8) is used. As the result, we have $m$-coupled differential equations in the parameter space $\left\{c_{\ell}\right\}$ when the loop equation (2.1) is expanded in the inverse power of $z$. This equation is called the flow equation.

$$
v_{k}\left(-\hbar^{2} \log Z_{N}^{(m)}\right)=d_{k} \quad \text { for } k=1, \cdots, m .
$$


Here $d_{k}$ is the coefficient obtained from the l.h.s. of (2.1) and is given in terms of the expectation values of the powers of $\lambda_{i}$ 's. Once we find the coefficients $d_{k}$ as an explicit function of $\left\{c_{\ell}\right\}$, we can find the partition function.

We will find $d_{k}$ in an explicit function of $c_{\ell}$ 's under the following framework. The parameters $c_{\ell}$ are defined so that the potential (1.8) has $m$-distinct saddle points $\left(z^{m+1} V^{\prime}(z)=0\right)$. Especially, we assume $c_{0}<0$ and $c_{\ell}$ 's $(\ell \geq 1)$ are alternating in sign in the ascending order in $\ell$ so that $c_{1}>0$. Also we assume a special hierarchical ordering of the parameters $\left|c_{k+1} / c_{k}\right| \ll\left|c_{k} / c_{k-1}\right|$. In terms of parameter $\eta_{k}=c_{k+1} c_{k-1} / c_{k}^{2}$, the hierarchical ordering shows that $\left|\eta_{k}\right| \ll 1$ and one may confirm that each saddle point is proportional to $c_{k+1} / c_{k}$ in the leading order of $\eta_{k}$.

In addition, one can demonstrate that the hierarchical ordering of the parameters corresponds to the special ordering of the position of vertex operators of the conformal block. To see this let us consider $m=2$ case. From the relation (1.3) one has $c_{0}=$ $\alpha_{0}+\alpha_{1}+\alpha_{2}, c_{1}=\alpha_{1} z_{1}+\alpha_{2} z_{2}$ and $c_{2}=\alpha_{0} z_{1} z_{2}$. Its solution has the form $z_{a}=\left(c_{1} / \alpha_{a}\right) x_{a}$ where $x_{1}=\left(1+\sqrt{1-4 \eta_{1} \alpha_{1} \alpha_{2} /\left(\alpha_{0} c_{0}\right)}\right) / 2$ and $x_{2}=\left(1-\sqrt{1-4 \eta_{1} \alpha_{1} \alpha_{2} /\left(\alpha_{0} c_{0}\right)}\right) / 2$. This shows that if one assumes $\left(\alpha_{1} \alpha_{2} / \alpha_{0}\right)$ is finite and small at the colliding limit, then one can expand $x_{a}$ 's in $\eta_{1}$ power series assuming $\left|\eta_{1}\right| \ll 1$,

$$
x_{1}=1+\mathcal{O}\left(\eta_{1}\right), \quad x_{2}=\eta_{1} \frac{\alpha_{1} \alpha_{2}}{\alpha_{0} c_{0}}\left(1+\mathcal{O}\left(\eta_{1}\right)\right) .
$$

It is worth to note that $x_{1}=\mathcal{O}(1)$ and $x_{2}=\mathcal{O}\left(\eta_{1}\right)$. In general, with the scaling of $z_{a}=c_{1} x_{a} / \alpha_{a}$, one has $x_{a}$ as $x_{a}=\mathcal{O}\left(\prod_{k=1}^{a-1} \eta_{k}\right)$. Therefore, the condition $\left|\eta_{k}\right| \ll 1$ is equivalent to put the positions of the primary fields with the hierarchy $\left|z_{m}\right| \ll\left|z_{m-1}\right| \ll$ $\cdots \ll\left|z_{2}\right| \ll\left|z_{1}\right| \rightarrow 0$.

\subsection{Inner product $\left\langle\Delta \mid I^{(m)}\right\rangle$}

Let us consider the simplest case $m=1$. The potential is given as $V(z)=-c_{0} \log (z)+c_{1} / z$ and has a stable equilibrium point on the positive axis of $z$ if $c_{0}<0$ and $c_{1}>0$. The partition function $Z_{N}^{(1)}$ is the function of $c_{0}$ and $c_{1}$ and the flow equation (2.3) has the form

$$
c_{1} \frac{\partial}{\partial c_{1}}\left(-\hbar^{2} \log Z_{N}^{(1)}\right)=d_{0}
$$

$d_{0}$ is simply obtained from the l.h.s. of $(2.1)$. The result is $d_{0}=h_{1}$ where we use the notation $h_{1}=\hbar b N\left(\hbar b N+2 c_{0}\right)$ for later convenience. From this information, one solves the equation (2.5) and gets the partition function $Z_{N}^{(1)}\left(c_{0}, c_{1}\right)=\left(c_{1}\right)^{-h_{1} / \hbar^{2}} Z\left(c_{0}\right)$ where $Z\left(c_{0}\right)$ is independent of $c_{1}$ but depends on $c_{0}$.

The flow equation, however, does not give any information on $c_{0}$ dependence. To see the $c_{0}$ dependence explicitly, one may rescale the integration variables $z=\xi c_{1}$ in the partition function $Z_{N}^{(1)}\left(c_{0}, c_{1}\right)$ to put the factorized into the form $Z_{N}^{(1)}\left(c_{0}, c_{1}\right)=\left(c_{1}\right)^{-h_{1} / \hbar^{2}} Z\left(c_{0}\right)$ where

$$
Z\left(c_{0}\right)=\int \prod_{i}^{N} d \xi_{i} \Delta^{2 \beta} e^{\frac{\sqrt{\beta}}{g} \sum_{i}\left(c_{0} \log \xi_{i}-1 / \xi_{i}\right)} .
$$


If the integration variable is inverted $\xi_{i}=1 / x_{i}$ this can be put in a more familiar form

$$
Z\left(c_{0}\right)=\int \prod_{i}^{N} d x_{i} \Delta^{2 \beta} e^{\frac{\sqrt{\beta}}{g} \sum_{i}\left(\alpha_{\infty} \log x_{i}-x_{i}\right)} .
$$

where $\alpha_{\infty}=-c_{0}-g(2+2 \beta(N-1)) / \sqrt{\beta}$. The integration variable is $x_{i} \geq 0$ and $\alpha_{\infty}$ is in the proper range so that the partition function is well-defined. Rescale $x_{i}$ by $\alpha_{\infty}$ and we have

$$
Z\left(c_{0}\right)=\left(\alpha_{\infty}\right)^{h_{1} / \hbar^{2}} \int \prod_{i}^{N} d x_{i} \Delta\left(x_{i}\right)^{2 \beta} e^{\frac{1}{g_{s}} \sum_{i}\left(\log \left(x_{i}\right)-x_{i}\right)}
$$

with $1 / g_{s}=\alpha_{\infty} \sqrt{\beta} / g$. When $\beta=1$, this partition function is the one considered by Penner [9] to describe the pseudo Euler characteristic.

The partition function $Z_{N}^{(1)}\left(c_{0}, c_{1}\right)$ is also obtained from the colliding limit of the 3 point conformal block directly. The 3 -point function is given by

$$
\mathcal{F}_{3}\left(\left\{e^{\alpha_{k} \phi\left(z_{k}\right)}\right\}\right)=\left|z_{0}-z_{1}\right|^{2 \gamma_{3}}\left|z_{1}-z_{\infty}\right|^{2 \gamma_{1}}\left|z_{\infty}-z_{0}\right|^{2 \gamma_{2}} C\left(\alpha_{0}, \alpha_{1}, \alpha_{\infty}\right)
$$

where $\gamma_{1}=\Delta_{0}-\Delta_{1}-\Delta_{\infty}, \gamma_{2}=\Delta_{1}-\Delta_{\infty}-\Delta_{0}, \gamma_{3}=\Delta_{\infty}-\Delta_{0}-\Delta_{1}$ and $C\left(\alpha_{0}, \alpha_{1}, \alpha_{\infty}\right)$ is a $z_{k}$-independent constant $[20,21]$. Putting $z_{0}=0, z_{\infty} \rightarrow \infty$ and $z_{1}=-c_{1} / \alpha_{0}$ and using $\alpha_{0}+\alpha_{1}+\alpha_{\infty}+b N=Q$, one has

$$
\left(z_{1}\right)^{2 \alpha_{0} \alpha_{1}} \mathcal{F}_{3}=\left(c_{1}\right)^{-h_{1} / \hbar^{2}}\left(-\alpha_{0}\right)^{h_{1} / \hbar^{2}} C\left(\alpha_{0}, \alpha_{1}, \alpha_{\infty}\right)
$$

up to an appropriate normalization. The power behavior of $c_{1}$ is the same as the one obtained from (2.5) and $Z\left(c_{0}\right)$ is identified as $Z\left(c_{0}\right)=\left(-\alpha_{0}\right)^{h_{1} / \hbar^{2}} C\left(\alpha_{0}, \alpha_{1}, \alpha_{\infty}\right)$ at the colliding limit $\alpha_{0}, \alpha_{1} \rightarrow \infty$ maintaining $c_{0}=\alpha_{0}+\alpha_{1}$ finite and $\alpha_{\infty}=Q-b N-c_{0}$. From now on, we do not bother to find the $c_{0}$ dependence which may be regarded as the normalization of the partition function. (Further comment is found in section 4).

When $m=2$, we have two flow equations.

$$
\begin{aligned}
\left(c_{1} \frac{\partial}{\partial c_{1}}+2 c_{2} \frac{\partial}{\partial c_{2}}\right)\left(-\hbar^{2} \log Z_{N}^{(2)}\right) & =d_{0} \\
c_{2} \frac{\partial}{\partial c_{1}}\left(-\hbar^{2} \log Z_{N}^{(2)}\right) & =d_{1}
\end{aligned}
$$

where $d_{0}=h_{1}$ as in the case $m=1 . \quad d_{1}=2 \hbar b N c_{1}+h_{2}\left\langle\sum_{i=1}^{N} \lambda_{i}\right\rangle$ is given in terms of expectation values $\left\langle\lambda_{i}\right\rangle$ and $h_{2}=2 \hbar b\left(\hbar b N+c_{0}\right)$. The equation (2.11) forces the partition function of the form

$$
-\hbar^{2} \log Z_{N}^{(2)}=h_{1} \log c_{1}+H^{(2)}\left(\eta_{1}\right)
$$

where $H^{(2)}\left(\eta_{1}\right)$ is the homogeneous solution. According to $(2.12), H^{(2)}\left(\eta_{1}\right)$ obeys

$$
2 \eta_{1}^{2} \frac{\partial}{\partial \eta_{1}} H^{(2)}\left(\eta_{1}\right)=h_{1} \eta_{1}-D_{1}
$$

where $D_{1} \equiv d_{1} c_{0} / c_{1}$ and its parametric dependence on $\eta_{1}$ is found using the filling fraction $N_{k}$

$$
\frac{\hbar b N_{k}}{2}=\oint_{\mathcal{A}_{k}} \frac{d z}{2 \pi i} W(z), \quad k=1,2
$$


where $\mathcal{A}_{k}$ is the contour loop ( $A$-cycle) which includes the eigenvalues around the saddle point. (We freely use the notation $N_{k}$ for the filling fraction instead of the ratio $N_{k} / N$ ). Putting the resolvent $2 W(z)=V^{\prime}(z)+\sqrt{V^{\prime}(z)^{2}+f(z)}$ from (2.1), one has parametric relation of the filling fraction

$$
\hbar b N_{k}=\oint_{\mathcal{A}_{k}} \frac{d z}{2 \pi i} \frac{\sqrt{\mathcal{P}_{4}(z)}}{z^{3}}
$$

where $\mathcal{P}_{4}(z)=\left(d_{0}+c_{0}^{2}\right) z^{4}+\left(d_{1}+2 c_{0} c_{1}\right) z^{3}+\left(c_{1}^{2}+2 c_{0} c_{2}\right) z^{2}+2 c_{1} c_{2} z+c_{2}^{2}$. One may assume that $\mathcal{P}_{4}(z)$ has four real and positive roots which need to be justified a posteriori. In this case, there are two branch cuts and branch points are the roots of $\mathcal{P}_{4}(z)=0$. For notational purpose, we denote the saddle point around $\left|c_{k} / c_{k-1}\right|$ as the $k$-th saddle point where the $k$-th cut and the filling fraction $N_{k}$ are associated.

The filling fraction (2.15) has a relation $N_{1}+N_{2}=N$ and therefore, $N_{1}$ is enough to find $D_{1}$. Integration is given in the elliptic function. But we will follow a practical way which works for $m \geq 2$. Using the integration variable $z=\xi c_{1} / c_{0}$, one has the rescaled $P_{4}(\xi)$ and the first cut is $\mathcal{O}\left(\eta_{1}^{0}\right)$. Therefore it is convenient to put $P_{4}(\xi)=\xi^{2} \tilde{\mathcal{P}}_{2}(\xi)+\mathcal{O}\left(\eta_{1}\right)$ where $\tilde{\mathcal{P}}_{2}(\xi)=\left(d_{0}+c_{0}^{2}\right) \xi^{2}+\left(D_{1}+2 c_{0}^{2}\right) \xi+c_{0}^{2}$. Therefore, the branch point is given by $\tilde{\mathcal{P}}_{2}(\xi)=0$ to the leading order of $\eta_{1}$, Expanding in powers of $\eta_{1}$,

$$
\hbar b N_{1}=\oint_{\mathcal{A}_{1}} \frac{d \xi}{2 \pi i}\left(\frac{\sqrt{\xi^{2} \tilde{\mathcal{P}}_{2}(\xi)}}{\xi^{3}}+\eta_{1} \frac{c_{0}^{2} \xi(\xi+1)}{\xi^{3} \sqrt{\xi^{2} \tilde{\mathcal{P}}_{2}(\xi)}}+\mathcal{O}\left(\eta_{1}^{2}\right)\right)
$$

one has the residue integration at $\xi=0$ and $\xi=\infty$.

$$
\hbar b N_{1}=\hbar b N-\frac{D_{1}}{2 c_{0}}+\eta_{1}\left(\hbar b N+\frac{(\hbar b N)^{2}-2 D_{1}}{2 c_{0}}-\frac{3 D_{1}^{2}}{8 c_{0}^{3}}\right)+\mathcal{O}\left(\eta_{1}^{2}\right) .
$$

Finding $D_{1}$ in small $\eta_{1}$ expansion, one has

$$
D_{1}=2 \hbar b N_{2} c_{0}+\eta_{1}\left(\hbar^{2} b^{2}\left(-2 N^{2}+6 N N_{1}-3 N_{1}^{2}\right)-2 \hbar b c_{0}\left(N-2 N_{1}\right)\right)+\mathcal{O}\left(\eta_{1}^{2}\right) .
$$

Equipped with the explicit $D_{1}$, we solve the equation (2.14) to find

$$
Z_{N}^{(2)}=\left(c_{1}\right)^{-h_{1} / \hbar^{2}}\left(\eta_{1}\right)^{-\frac{b N_{2}}{2}\left(3 b N_{2}+4 c_{0} / \hbar\right)} e^{-\frac{b N_{2} c_{0} / \hbar}{\eta_{1}}+\mathcal{O}\left(\eta_{1}\right)}
$$

The partition function $Z_{N}^{(2)}$ has an interesting feature of the singularity in $\eta_{1}$. The term with the factor $N_{2}^{2}$ comes from the Vandermonde determinant. On the other hand, the term with factor linear in $N_{2}$ has the essential singularity of the form $\exp \left(1 / \eta_{1}\right)$ which is proportional to the energy difference of the two saddle points which is the instanton effect $[22,23]$. Suppose we put $N_{2}=0$ which has no instanton at all. Then, the partition function becomes regular in $\eta_{1}, Z_{N}^{(2)}=\left(c_{1}\right)^{-h_{1} / \hbar^{2}}\left(1+\mathcal{O}\left(\eta_{1}\right)\right)$ and if one puts $\eta_{1} \rightarrow 0$, the partition function reduces to $Z_{N}^{(1)}$, the one with $m=1$. In other words, when $N_{2}=0$, the partition function $Z_{N}^{(2)}$ has the smooth limit to $Z_{N}^{(1)}$ if $\eta_{1} \rightarrow 0$ because the singular dependence of $\eta_{1}$ disappears. This limiting procedure is very general as can be found below for the case with $m=3$. 


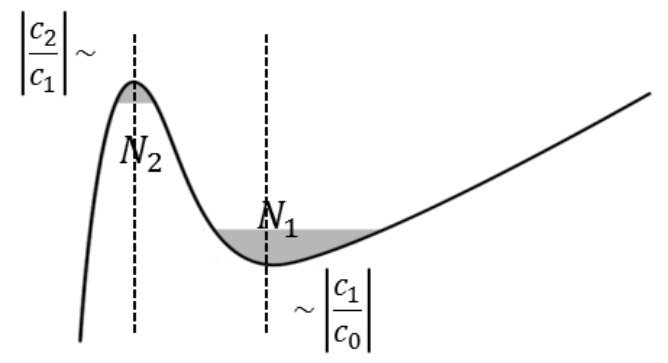

Figure 3. Shape of $m=2$ potential

When $m=3$, we have three flow equations,

$$
\begin{aligned}
\left(c_{1} \frac{\partial}{\partial c_{1}}+2 c_{2} \frac{\partial}{\partial c_{2}}+3 c_{3} \frac{\partial}{\partial c_{3}}\right)\left(-\hbar^{2} \log Z_{N}^{(3)}\right) & =d_{0} \\
\left(c_{2} \frac{\partial}{\partial c_{1}}+2 c_{3} \frac{\partial}{\partial c_{2}}\right)\left(-\hbar^{2} \log Z_{N}^{(3)}\right) & =d_{1} \\
c_{3} \frac{\partial}{\partial c_{1}}\left(-\hbar^{2} \log Z_{N}^{(3)}\right) & =d_{2}
\end{aligned}
$$

where $d_{0}=h_{1}$ and $d_{1}=2 \hbar b N c_{1}+h_{2}\left\langle\sum_{i=1}^{N} \lambda_{i}\right\rangle$ as found in (2.11) and (2.12). $d_{2}$ is new and $d_{2}=2 \hbar b N c_{2}+2 \hbar b c_{1}\left\langle\sum_{i=1}^{N} \lambda_{i}\right\rangle+\hbar^{2} b^{2}\left\langle\sum_{i=1}^{N} \lambda_{i}\right\rangle^{2}+h_{2}\left\langle\sum_{i=1}^{N} \lambda_{i}^{2}\right\rangle$.

According to (2.21), one puts the partition function of the form

$$
-\hbar^{2} \log Z_{N}^{(3)}=h_{1} \log c_{1}+H^{(3)}\left(\eta_{1}, \eta_{2}\right)
$$

where $H^{(3)}\left(\eta_{1}, \eta_{2}\right)$ is the homogeneous solution and is written as the function of $\eta_{1}=c_{0} c_{2} / c_{1}^{2}$ and $\eta_{2}=c_{1} c_{3} / c_{2}^{2}$. The differential equation of $H^{(3)}\left(\eta_{1}, \eta_{2}\right)$ is obtained from the rest of the equations (2.22) and (2.23)

$$
\begin{aligned}
\frac{\partial H^{(3)}}{\partial \eta_{1}} & =\frac{D_{2}-\left(D_{1}+4 D_{2}\right) \eta_{2}+4 d_{0} \eta_{1} \eta_{2}^{2}}{6 \eta_{1}^{2} \eta_{2}^{2}} \\
\frac{\partial H^{(3)}}{\partial \eta_{2}} & =\frac{D_{2}-\left(D_{1}+D_{2}\right) \eta_{2}+d_{0} \eta_{1} \eta_{2}^{2}}{3 \eta_{1} \eta_{2}^{3}}
\end{aligned}
$$

where $D_{1} \equiv d_{1} c_{0} / c_{1}$ and $D_{2} \equiv d_{2} c_{0} / c_{2}$ are fixed by the filling fraction

$$
\hbar b N_{k}=\oint_{\mathcal{A}_{k}} \frac{d z}{2 \pi i} \sqrt{\frac{\mathcal{P}_{6}(z)}{z^{8}}}, \quad k=1,2,3
$$

where $N=N_{1}+N_{2}+N_{3}$ and $\mathcal{P}_{6}(z)=\left(d_{0}+c_{0}^{2}\right) z^{6}+\left(d_{1}+2 c_{0} c_{1}\right) z^{5}+\left(c_{1}^{2}+2 c_{0} c_{2}+d_{2}\right) z^{4}+$ $2\left(c_{1} c_{2}+c_{0} c_{3}\right) z^{3}+\left(c_{2}^{2}+2 c_{1} c_{3}\right) z^{2}+2 c_{2} c_{3} z+c_{3}^{2}$.

The contour integration $\mathcal{A}_{1}$ (figure $5(\mathrm{a})$ ) is around $z=\left|c_{1} / c_{0}\right|$. We may rescale $z=$ $\xi c_{1} / c_{0}$ so that $\left(c_{0} / c_{1}\right)^{6} \mathcal{P}_{6}\left(\xi c_{1} / c_{0}\right)=\xi^{4} \tilde{\mathcal{P}}_{2}(\xi)+\eta_{1} \xi^{3}\left(\left(D_{2}+2 c_{0}^{2}\right) \xi+2 c_{0}^{2}\right)+\mathcal{O}\left(\eta_{1}^{2}\right)$ where $\tilde{\mathcal{P}}_{2}(\xi)=\left(d_{0}+c_{0}^{2}\right) \xi^{2}+\left(D_{1}+2 c_{0}^{2}\right) \xi+c_{0}^{2}$. Here $\xi, D_{1}$ and $D_{2}$ are assumed $\mathcal{O}(1)$. Expanding the filling fraction $N_{1}$ in powers of $\eta_{1}$ and $\eta_{2}$, one has

$$
\hbar b N_{1}=\oint_{\mathcal{A}_{1}} \frac{d \xi}{2 \pi i}\left(\frac{\sqrt{\xi^{4} \tilde{\mathcal{P}}_{2}(\xi)}}{\xi^{4}}+\eta_{1} \frac{\left(D_{2}+2 c_{0}^{2}\right) \xi+2 c_{0}^{2}}{2 \xi^{3} \sqrt{\tilde{\mathcal{P}}_{2}(\xi)}}+\mathcal{O}\left(\eta_{1}^{2}\right)\right) .
$$




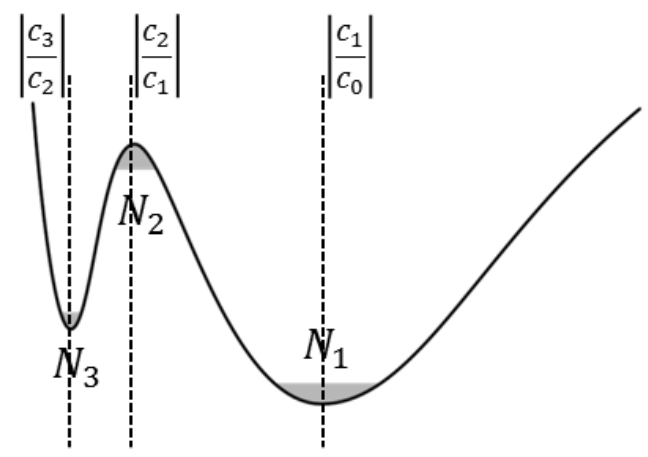

Figure 4. Shape of $m=3$ potential

(a)

$\xi$

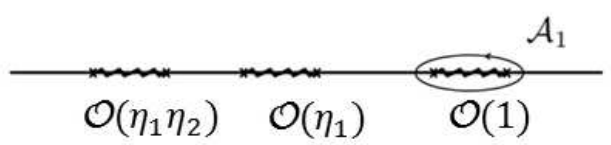

(b)
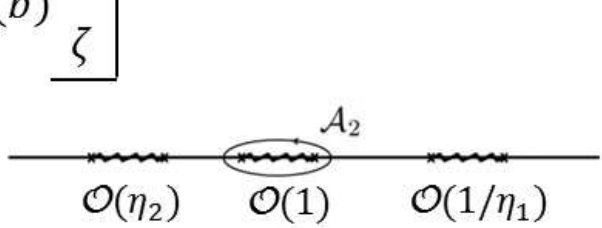

Figure 5. Contours for the first(a) and second(b) cut

The residues at $\xi=0$ and $\xi=\infty$ give the contour contribution

$$
\hbar b N_{1}=\hbar b N-\frac{D_{1}}{2 c_{0}}+\eta_{1} \frac{4 c_{0}^{2}\left(h_{1}-2 D_{1}+D_{2}\right)+D_{1}\left(2 D_{2}-3 D_{1}\right)}{8 c_{0}^{3}}+\mathcal{O}\left(\eta_{1}^{2}\right) .
$$

The second cut lies around $z=\left|c_{2} / c_{1}\right|$. We rescale $z=\zeta c_{2} / c_{1}$ for the contour integrations $\mathcal{A}_{2}$ (see figure $5(\mathrm{~b})$ ) and find the polynomial $\eta_{1}^{2}\left(c_{1} / c_{2}\right)^{6} \mathcal{P}_{6}\left(\zeta c_{2} / c_{1}\right)=$ $c_{0}^{2} \zeta^{2}(\zeta+1)^{2}+2 \eta_{2} c_{0}^{2} \zeta(\zeta+1)+\eta_{2}^{2} c_{0}^{2}+\eta_{1}\left\{\zeta^{4}\left(\left(2 c_{0}^{2}+D_{1}\right) \zeta+2 c_{0}^{2}+D_{2}\right)+\eta_{2}\left(2 c_{0}^{2} \zeta^{3}\right)\right\}+\eta_{1}^{2} \zeta^{6}\left(d_{0}+c_{0}^{2}\right)$ where the dominant part is the squared form $\left(\zeta^{2}+\zeta\right)^{2}$. Therefore, the small $\eta_{1}$ and $\eta_{2}$ expansion has no branch cut integral. After integration, one has

$$
\begin{aligned}
\hbar b N_{2}= & \frac{1}{2 c_{0}}\left(D_{1}-D_{2}+\left(D_{1}-2 D_{2}\right) \eta_{2}+3\left(D_{1}-2 D_{2}\right) \eta_{2}^{2}+\mathcal{O}\left(\eta_{2}^{3}\right)\right) \\
& +\eta_{1}\left[\frac{D_{1}\left(3 D_{1}-2 D_{2}\right)-4 c_{0}^{2}\left(h_{1}-2 D_{1}+D_{2}\right)}{8 c_{0}^{3}}\right. \\
& \left.+\frac{3 D_{2}^{2}-2 c_{0}^{2}\left(h_{1}-3 D_{2}\right)}{4 c_{0}^{3}} \eta_{2}^{2}+\mathcal{O}\left(\eta_{2}^{3}\right)\right]+\mathcal{O}\left(\eta_{1}^{2}\right) .
\end{aligned}
$$

$D_{1}$ and $D_{2}$ are obtained from equations (2.29) and (2.30)

$$
\begin{aligned}
D_{1}= & 2 \hbar b c_{0}\left(N-N_{1}\right)+\eta_{1}\left[A_{1}+A_{2} \eta_{2}+\mathcal{O}\left(\eta_{2}^{2}\right)\right]+\mathcal{O}\left(\eta_{1}^{2}, \eta_{2}^{3}\right) \\
D_{2}= & 2 \hbar b c_{0} N_{3}+\eta_{2}\left(2 \hbar b c_{0}\left(N_{2}-N_{3}\right)\right)+\eta_{2}^{2}\left(2 \hbar b c_{0}\left(N_{2}-N_{3}\right)\right) \\
& +\eta_{1}\left[A_{1} \eta_{2}+B_{1} \eta_{2}^{2}+\mathcal{O}\left(\eta_{2}^{3}\right)\right]+\mathcal{O}\left(\eta_{1}^{3}, \eta_{2}^{3}\right)
\end{aligned}
$$

where $A_{1}=-2 \hbar b c_{0}\left(N_{2}-N_{1}\right)-2 \hbar^{2} b^{2} N\left(N_{2}-N_{1}\right)-\hbar^{2} b^{2} N_{1}\left(-2 N_{2}+N_{1}\right), A_{2}=\left(-2 \hbar b c_{0}-\right.$ $\left.2 \hbar^{2} b^{2}\left(N-N_{1}\right)\right)\left(N_{3}-N_{2}\right)$, and $B_{1}=2 \hbar b c_{0}\left(N_{3}-N_{2}\right)+\hbar^{2} b^{2}\left(3 N^{2}+6 N_{2}^{2}+10 N_{1} N_{2}+3 N_{1}^{2}-\right.$ 
$\left.2 N\left(3 N_{1}+5 N_{2}\right)\right)$. Plugging $D_{1}$ and $D_{2}$ into a system of equations (2.25) and (2.26), we have the partition function

$$
\begin{aligned}
-\hbar^{2} \log Z_{N}^{(3)}= & h_{1} \log c_{1}-\frac{\hbar b c_{0} N_{3}}{3 \eta_{1} \eta_{2}^{2}}+\frac{2 \hbar b c_{0} N_{3}}{\eta_{1} \eta_{2}}+\frac{\hbar b c_{0}\left(N_{2}-N_{3}\right)}{\eta_{1}} \\
& +\frac{\hbar b}{2}\left(4 c_{0}\left(N-N_{1}\right)+\hbar b\left(3 N_{2}^{2}+4 N_{2} N_{3}+3 N_{3}^{2}\right)\right) \log \eta_{1} \\
& +2 \hbar b N_{3}\left(\hbar b N_{3}+c_{0}\right) \log \eta_{2}+\mathcal{O}\left(\eta_{1}, \eta_{2}\right)
\end{aligned}
$$

or

$$
\begin{aligned}
Z_{N}^{(3)}= & \left(c_{1}\right)^{-h_{1} / \hbar^{2}}\left(\eta_{1}\right)^{-\frac{b}{2}\left(N_{2}\left(3 b N_{2}+4 c_{0} / \hbar\right)+N_{3}\left(3 b N_{3}+4 c_{0} / \hbar\right)+4 b N_{2} N_{3}\right)}\left(\eta_{2}\right)^{-2 b N_{3}\left(b N_{3}+c_{0} / \hbar\right)} \\
& e^{-\frac{b c_{0}}{\eta_{1} \hbar}\left(\left(N_{2}-N_{3}\right)+\frac{2 N_{3}}{\eta_{2}}-\frac{N_{3}}{3 \eta_{2}^{2}}\right)+\mathcal{O}\left(\eta_{1}, \eta_{2}\right)}
\end{aligned}
$$

Note that the term linear in $N_{3}$ shows the non-trivial instanton effect whose exact contribution is not easy to calculate in other ways. When $N_{3}=0$, the partition function has the smooth limit $Z_{N}^{(m=2)}$ as $\eta_{2} \rightarrow 0$.

\subsection{Inner product $\left\langle I^{(n)} \mid I^{(m)}\right\rangle$}

Let us now consider the colliding limit of the conformal block as shown in figure 6 . In this limit, the partition function $Z_{N}^{(n ; m)}$ has the potential $V(z)=-c_{0} \log z+V_{-}(z)+V_{+}(z)$

$$
V_{-}(z)=\sum_{s=1}^{m} \frac{c_{s}}{s z^{s}}, \quad V_{+}(z)=-\sum_{t=1}^{n} \frac{c_{-t} z^{t}}{t} .
$$

The potential is modified to have a new Liouville momentum $c_{0}=\sum_{s=0}^{m} \alpha_{s}$ at zero and $c_{\infty}=\alpha_{\infty}+\sum_{t=m+1}^{m+n} \alpha_{t}$ at infinity so that the neutrality condition is $c_{0}+c_{\infty}+\hbar b N=$ $\hbar Q$. The additional positive power term in $V_{+}(z)$ characterizes the irregular singularity at infinity. Thus, this matrix model is identified with the inner product $\left\langle I^{(n)} \mid I^{(m)}\right\rangle$ between the irregular vector of the rank $n$ at infinity and irregular vector of the rank $m$ at zero.

The quantum correction $f(z)=\sum_{k} d_{k} / z^{2+k}$ has $d_{k}=0$ when $k \geq m$ or $k \leq-(n+1)$ and $d_{-n}=2 \hbar b N c_{-n}$. The remaining terms are given as the $(m+n-1)$ flow equations

$$
\begin{aligned}
& d_{k}=v_{k}\left(-\hbar^{2} \log Z_{N}^{(n ; m)}\right), \quad v_{k}=\sum_{s=1}^{m} s c_{s+k} \frac{\partial}{\partial c_{s}} \quad \text { when } 0 \leq k \leq m-1 \\
& d_{-k}=2 \hbar b N c_{-k}+u_{k}\left(-\hbar^{2} \log Z_{N}^{(n ; m)}\right), \quad u_{k}=\sum_{t=1}^{n} t c_{-t-k} \frac{\partial}{\partial c_{-t}} \quad \text { when } 1 \leq k \leq n-1 .
\end{aligned}
$$

If one defines $\varphi(z) \equiv V^{\prime}(z)^{2}+f(z)$, one has $\varphi(z)=\sum_{k=-2 n}^{2 m} \Lambda_{k} / z^{k+2}$ where $\Lambda_{k}=$ $d_{k}+\sum_{k=\ell+\ell^{\prime}} c_{\ell} c_{\ell^{\prime}}$. Regarding $\varphi(z)$ as the expectation value of the energy momentum tensor $\varphi(z)=\left\langle I^{(n)}|T(z)| I^{(m)}\right\rangle /\left\langle I^{(n)} \mid I^{(m)}\right\rangle$, one has the eigenstate at origin

$$
L_{k}\left|I^{(m)}\right\rangle=\Lambda_{k}\left|I^{(m)}\right\rangle \quad \text { when } m \leq k \leq 2 m
$$




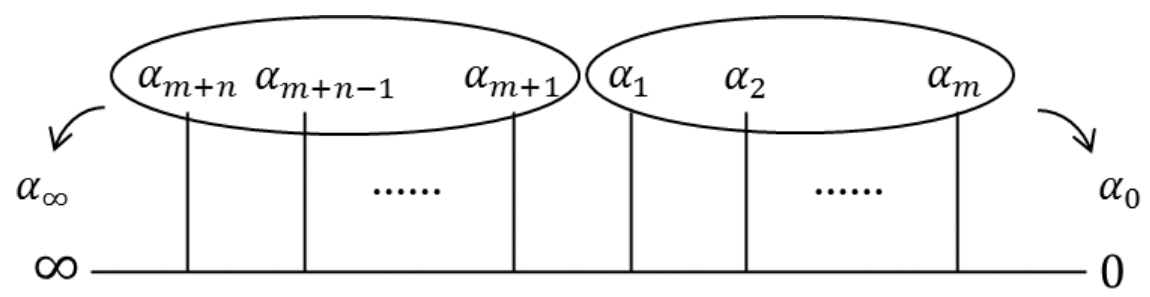

Figure 6. Schematic diagram of $\left\langle I^{(n)} \mid I^{(m)}\right\rangle$

and another at infinity

$$
\left\langle I^{(n)}\right| L_{k}=\Lambda_{k}\left\langle I^{(n)}\right| \quad \text { when }-2 n \leq k \leq-n .
$$

This definition is consistent with the conjugate of Virasoro generators $L_{-k}=L_{k}^{\dagger}$.

We assume $\eta_{k} \ll 1$ for $-(n-1) \leq k \leq m-1$ which ensures that each saddle point is proportional to $\left|c_{k+1} / c_{k}\right|$. Suppose we rescale the integral variables $\lambda_{i}$ as $\lambda_{i} c_{0} / c_{-1}$, the partition function has the form

$$
Z_{N}^{(n ; m)}=\left(\frac{c_{-1}}{c_{0}}\right)^{\left(h_{1} / \hbar^{2}-b N Q\right)} \int\left[\prod_{i=1}^{N} d \lambda_{i}\right] \Delta\left(\lambda_{i}\right)^{2 \beta} \exp \left(-\frac{\sqrt{\beta}}{g} \sum_{i} \hat{V}\left(\lambda_{i}\right)\right)
$$

where $\hat{V}(z)=-c_{0} \log z+V_{+}\left(z c_{0} / c_{-1}\right)+V_{-}\left(z c_{0} / c_{-1}\right)$. Note that $-b N Q$ power of $c_{-1}$ is the sub-leading contribution. In the large $N$ expansion, we have

$$
-\hbar^{2} \log Z_{N}^{(n ; m)}=-h_{1} \log \left(c_{-1} / c_{0}\right)+H^{(n ; m)}\left(\left\{\eta_{k}\right\}\right)
$$

where $H^{(n ; m)}$ is the function of $\eta_{k}$ 's because the scaled potential has the coefficient $c_{s}\left(c_{0} / c_{-1}\right)^{-s}=c_{0} \prod_{k=0}^{s-1} \eta_{k}^{s-k}$ and $c_{-t}\left(c_{0} / c_{-1}\right)^{t}=c_{0} \prod_{j=1}^{t-1} \eta_{-j}^{t-j}$.

The partition function can be evaluated as done in $\left\langle\Delta \mid I^{(m)}\right\rangle$. To find $d_{k}$ as the functions of $\eta_{k}$ 's we use the filling fraction relations $\left(N=\sum_{k=-(n-1)}^{m} N_{k}\right)$

$$
\hbar b N_{k}=\oint_{\mathcal{A}_{k}} \frac{d z}{2 \pi i} \sqrt{\varphi(z)}=\oint_{\mathcal{A}_{k}} \frac{d z}{2 \pi i} \frac{\sqrt{\mathcal{P}_{2(m+n)}(z)}}{z^{m+1}} .
$$

$\mathcal{P}_{2(m+n)}(z)$ is a polynomial of order $2(m+n)$ and the integrand has $(m+n)$ cuts. Integrating over $\mathcal{A}_{k}$ is done after rescaling $z$ to $\xi c_{k} / c_{k-1}$ so that the $k$-th cut is $\mathcal{O}\left(\left\{\eta_{k}^{0}\right\}\right)$ in $\xi$-plane. On the other hand, $(k+a)$-th cut with $a \geq 1$ goes to zero, $\mathcal{O}\left(\prod_{\ell=0}^{a-1} \eta_{k+\ell}\right)$ and $(k-a)$-th cut to infinity, $\mathcal{O}\left(\prod_{\ell=1}^{a} 1 / \eta_{k-\ell}\right)$ as $\left\{\eta_{j}\right\} \rightarrow 0$.

The simplest example is $n=m=1$. It is convenient to put $Z_{N}^{(1 ; 1)}$ in the form $(2.42)$ whose flow equation is

$$
\eta_{0} \frac{\partial}{\partial \eta_{0}} H^{(1 ; 1)}\left(\eta_{0}\right)=d_{0}
$$

Filling fraction integral has $\mathcal{P}_{4}(z)=c_{-1}^{2} z^{4}+2 c_{-1}\left(\hbar b N+c_{0}\right) z^{3}+\left(2 c_{1} c_{-1}+d_{0}+c_{0}^{2}\right) z^{2}+2 c_{1} c_{0} z+$ $c_{1}^{2}$. Rescaling $z$ to $\xi c_{0} / c_{-1}$ we have $\left(c_{-1} / c_{0}\right)^{2} \mathcal{P}_{4}\left(\xi c_{0} / c_{-1}\right)=\xi^{2} \mathcal{P}_{2}(\xi)+\eta_{0}\left[2 c_{0}^{2} \xi(\xi+1)\right]+\eta_{0}^{2} c_{0}^{2}$ 
where $\mathcal{P}_{2}(\xi)=c_{0}^{2} \xi^{2}+2 c_{0}\left(\hbar b N+c_{0}\right) z+\left(d_{0}+c_{0}^{2}\right)$. After this we have $\hbar b N_{0}=\hbar b N+c_{0}-$ $\sqrt{d_{0}+c_{0}^{2}}+\mathcal{O}\left(\eta_{0}\right)$ or $d_{0}=\hbar b N_{1}\left(\hbar b N_{1}+2 c_{0}\right)+\mathcal{O}\left(\eta_{0}\right)$. Thus the partition function is given as

$$
-\hbar^{2} \log Z_{N}^{(1 ; 1)}=-h_{1} \log \left(c_{-1} / c_{0}\right)+\hbar b N_{1}\left(\hbar b N_{1}+2 c_{0}\right) \log \eta_{0}+\mathcal{O}\left(\eta_{0}\right) .
$$

When $n=1$ and $m=2$, we have two flow equations

$$
\begin{aligned}
\eta_{0} \frac{\partial}{\partial \eta_{0}} H^{(1 ; 2)}\left(\eta_{0}, \eta_{1}\right) & =d_{0} \\
\left(\eta_{0} \eta_{1} \frac{\partial}{\partial \eta_{0}}-2 \eta_{1}^{2} \frac{\partial}{\partial \eta_{1}}\right) H^{(1 ; 2)}\left(\eta_{0}, \eta_{1}\right) & =D_{1}
\end{aligned}
$$

where $D_{1} \equiv d_{1} c_{0} / c_{1}$. Using the polynomial $\mathcal{P}_{6}(z)=c_{-1}^{2} z^{6}+\left(d_{-1}+2 c_{-1} c_{0}\right) z^{5}+\left(2 c_{1} c_{-1}+\right.$ $\left.d_{0}+c_{0}^{2}\right) z^{4}+\left(2 c_{2} c_{-1}+d_{1}+2 c_{1} c_{0}\right) z^{3}+\left(c_{1}^{2}+2 c_{2} c_{0}\right) z^{2}+2 c_{1} c_{2} z+c_{2}^{2}$, we have

$$
\begin{aligned}
& \hbar b N_{0}=\hbar b N+c_{0}-\sqrt{d_{0}+c_{0}^{2}}+\mathcal{O}\left(\eta_{0}\right) \\
& \hbar b N_{1}=-\frac{D_{1}}{2 c_{0}}-c_{0}+\sqrt{d_{0}+c_{0}^{2}}+\eta_{1}\left(4 c_{0}^{2}\left(d_{0}-2 D_{1}\right)-3 D_{1}^{2}\right) /\left(8 c_{0}^{3}\right)+\mathcal{O}\left(\eta_{1}{ }^{2}\right)
\end{aligned}
$$

Inverting the relations, we have $d_{0}=\hbar b\left(N-N_{0}\right)\left(\hbar b\left(N-N_{0}\right)+2 c_{0}\right)+\mathcal{O}\left(\eta_{0}\right)$ and $D_{1}=$ $2 \hbar b c_{0} N_{2}+\eta_{1}\left[\hbar^{2} b^{2}\left(N^{2}-3 N_{2}^{2}-2 N N_{0}+N_{0}^{2}\right)+2\left(N_{1}-N_{2}\right) c_{0}\right]+\mathcal{O}\left(\eta_{1}^{2}\right)$. Therefore,

$$
\begin{aligned}
H^{(1 ; 2)}\left(\eta_{0}, \eta_{1}\right)= & \frac{\hbar b c_{0} N_{2}}{\eta_{1}}+\hbar b\left(N_{1}+N_{2}\right)\left(\hbar b\left(N_{1}+N_{2}\right)+2 c_{0}\right) \log \eta_{0} \\
& +\frac{\hbar b N_{2}}{2}\left(3 \hbar b N_{2}+4 c_{0}\right) \log \eta_{1}+\mathcal{O}\left(\eta_{0}, \eta_{1}\right)
\end{aligned}
$$

or

$$
Z_{N}^{(1 ; 2)}=\left(c_{-1} / c_{0}\right)^{h_{1} / \hbar^{2}}\left(\eta_{0}\right)^{-b\left(N_{1}+N_{2}\right)\left(b\left(N_{1}+N_{2}\right)+2 \frac{c_{0}}{\hbar}\right)}\left(\eta_{1}\right)^{-\frac{b N_{2}}{2}\left(3 b N_{2}+4 \frac{c_{0}}{\hbar}\right)} e^{-\frac{b N_{2} c_{0} / \hbar}{\eta_{1}}+\mathcal{O}\left(\eta_{0}, \eta_{1}\right)} .
$$

As $m$ and $n$ increase, the flow equations becomes more complicated. We give the explicit result for $n=m=2$ for later comparison. We have three flow equations

$$
\begin{aligned}
& \frac{\partial H^{(2 ; 2)}}{\partial \eta_{0}}=\frac{d_{0}}{\eta_{0}} \\
& \frac{\partial H^{(2 ; 2)}}{\partial \eta_{1}}=\frac{d_{0} \eta_{1}-D_{1}}{2 \eta_{1}^{2}} \\
& \frac{\partial H^{(2 ; 2)}}{\partial \eta_{-1}}=\frac{d_{0} \eta_{-1}-D_{-1}-h_{1} \eta_{-1}+2 \hbar b N c_{0}}{2 \eta_{-1}^{2}} .
\end{aligned}
$$

where $D_{-1} \equiv d_{-1} c_{0} / c_{-1}$. Using the polynomial

$$
\begin{aligned}
\mathcal{P}_{8}(z)= & c_{-2}^{2} z^{8}+2 c_{-1} c_{-2} z^{7}+\left(c_{-1}^{2}+2 c_{-2}\left(\hbar b N+c_{0}\right)\right) z^{6}+\left(2 c_{1} c_{-2}+d_{-1}+2 c_{-1} c_{0}\right) z^{5} \\
& +\left(2 c_{1} c_{-1}+2 c_{2} c_{-2}+d_{0}+c_{0}^{2}\right) z^{4}+\left(2 c_{2} c_{-1}+d_{1}+2 c_{1} c_{0}\right) z^{3} \\
& +\left(c_{1}^{2}+2 c_{2} c_{0}\right) z^{2}+2 c_{1} c_{2} z+c_{2}^{2}
\end{aligned}
$$


the filling fractions are evaluated

$$
\begin{aligned}
\hbar b N_{0}= & \frac{D_{-1}}{2 c_{0}}+c_{0}-\sqrt{d_{0}+c_{0}^{2}} \\
& +\frac{-4 c_{0}^{2}\left(d_{0}-2 D_{-1}\right)+3 D_{-1}^{2}-4 \hbar b N D_{-1} c_{0}-8 \hbar b N c_{0}^{3}}{8 c_{0}^{3}} \eta_{-1}+\mathcal{O}\left(\eta_{0}, \eta_{-1}^{2}\right) \\
\hbar b N_{1}= & -\frac{D_{1}}{2 c_{0}}-c_{0}+\sqrt{d_{0}+c_{0}^{2}}+\frac{4 c_{0}^{2}\left(d_{0}-2 D_{1}\right)-3 D_{1}^{2}}{8 c_{0}^{3}} \eta_{1}+\mathcal{O}\left(\eta_{0}, \eta_{1}^{2}\right) \\
\hbar b N_{2}= & \frac{D_{1}}{2 c_{0}}+\frac{-4 c_{0}^{2}\left(d_{0}-2 D_{1}\right)+3 D_{1}^{2}}{8 c_{0}^{3}}+\mathcal{O}\left(\eta_{0}, \eta_{1}^{2}\right)
\end{aligned}
$$

Inverting the result, one has $d_{0}=A_{1} D_{1}=B_{1}+B_{2} \eta_{1}$ and $D_{-1}=C_{1}+C_{2} \eta_{-1}$ where

$$
\begin{aligned}
& A_{1}=\hbar b\left(N_{1}+N_{2}\right)\left(\hbar b\left(N_{1}+N_{2}\right)+2 c_{0}\right), \quad B_{1}=2 \hbar b c_{0} N_{2}, C_{1}=2 \hbar b c_{0}\left(N_{0}+N_{1}+N_{2}\right) \\
& B_{2}=\hbar b\left[\hbar b\left(N_{1}^{2}+2 N_{1} N_{2}-2 N_{2}^{2}\right)+2\left(N_{1}-N_{2}\right) c_{0}\right] \\
& \begin{aligned}
C_{2}= & \hbar b\left[2 \hbar b N\left(N-N_{-1}\right)-3 \hbar b\left(N-N_{-1}\right)^{2}\right. \\
\quad & \left.\quad+\left(N_{1}+N_{2}\right)\left(\hbar b\left(N_{1}+N_{2}\right)-2 c_{0}\right)-4 c_{0}\left(N-N_{-1}\right)+2 N c_{0}\right]
\end{aligned}
\end{aligned}
$$

and one has the partition function

$$
\begin{aligned}
H^{(2 ; 2)}\left(\eta_{-1}, \eta_{0}, \eta_{1}\right)=-\frac{\hbar b c_{0} N_{-1}}{\eta_{-1}}+\frac{\hbar b c_{0} N_{2}}{\eta_{1}}+\hbar b\left(N_{1}+N_{2}\right)\left(\hbar b\left(N_{1}+N_{2}\right)+2 c_{0}\right) \log \eta_{0} \\
+\frac{\hbar b N_{2}\left(3 \hbar b N_{2}+4 c_{0}\right)}{2} \log \eta_{1}-\frac{\hbar b N_{-1}\left(-3 \hbar b N_{-1}+4\left(c_{0}+\hbar b N\right)\right)}{2} \log \eta_{-1} \\
\quad+\mathcal{O}\left(\eta_{-1}, \eta_{0}, \eta_{1}\right)
\end{aligned}
$$

or

$$
\begin{array}{r}
Z_{N}^{(2 ; 2)}=\left(c_{-1} / c_{0}\right)^{h_{1} / \hbar^{2}}\left(\eta_{0}\right)^{-b\left(N_{1}+N_{2}\right)\left(b\left(N_{1}+N_{2}\right)+2 c_{0} / \hbar\right)}\left(\eta_{-1}\right)^{\frac{b N_{-1}}{2}\left(-3 b N_{-1}+4\left(c_{0} / \hbar+b N\right)\right)} \\
\left(\eta_{1}\right)^{-\frac{b N_{2}}{2}\left(3 b N_{2}+4 c_{0} / \hbar\right)} e^{-\frac{b N_{2} c_{0} / \hbar}{\eta_{1}}+\frac{b N_{-1} c_{0} / \hbar}{\eta_{-1}}+\mathcal{O}\left(\eta_{-1}, \eta_{0}, \eta_{1}\right)} .
\end{array}
$$

\section{Hierarchical structure of the partition function}

It should be noted that given $N_{2}$, the inner products $\left\langle I^{(1)} \mid I^{(2)}\right\rangle$ in (2.49), $\left\langle I^{(2)} \mid I^{(2)}\right\rangle$ in (2.57) and $\left\langle\Delta \mid I^{(2)}\right\rangle$ in $(2.20)$ have the same $\eta_{1}$ dependence. Likewise given $N_{1}$ and $N_{2}$, one can see the same $\eta_{0}$ dependence in $\left\langle I^{(2)} \mid I^{(2)}\right\rangle$ and $\left\langle I^{(1)} \mid I^{(2)}\right\rangle$. From this observation, one may wonder if how much singular structure in $\left\langle I^{(n)} \mid I^{(m)}\right\rangle$ is shared with others. We will investigate the possibility of this singular structure in detail and present a way to find the partition function without using the filling fraction contour integration.

\subsection{Singular structure in $\left\langle I^{(n)} \mid I^{(m)}\right\rangle$ and $\left\langle\Delta \mid I^{(m)}\right\rangle$}

Let us investigate the small $\eta_{0}$-behavior in the inner product $\left\langle I^{(n)} \mid I^{(m)}\right\rangle$. First we rearrange the integration variables in two groups. One group is the integration variables which lies 
around the $k$-th saddle point with $k \geq 1$ whose integration variables are denoted as $\lambda_{i}^{L}>0$. The number of eigenvalues is $N_{L}$. The other group is denoted as $\lambda_{i}^{R}>0$ which lives around the $k$-th saddle point with $k \leq 0$. The number of $\lambda_{i}^{R}$ is $N_{R}$. The partition function $Z_{N}^{(n ; m)}$ is rewritten in terms of two regrouped variables

$$
\begin{aligned}
Z_{N}^{(n ; m)}=\int\left[\prod_{i=1}^{N_{L}} d \lambda_{i}^{L}\right]\left[\prod_{j=1}^{N_{R}} d \lambda_{j}^{R}\right] \Delta\left(\lambda^{L}\right)^{2 \beta} \Delta\left(\lambda^{R}\right)^{2 \beta} \prod_{i=1}^{N_{L}} \prod_{j=1}^{N_{R}}\left(\lambda_{j}^{R}-\lambda_{i}^{L}\right)^{2 \beta} \\
\\
\times \exp \left[-\frac{\sqrt{\beta}}{g}\left(\sum_{i=1}^{N_{L}} V\left(\lambda_{i}^{L}\right)+\sum_{j=1}^{N_{R}} V\left(\lambda_{j}^{R}\right)\right)\right]
\end{aligned}
$$

whose potential is given in (2.36). If we use $\eta_{0} \ll 1$, we may put $Z_{N}^{(n ; m)}$ into the factorized form $Z_{L} Z_{R}\left(1+\mathcal{O}\left(\eta_{0}\right)\right)$. The reason is as follows. The integration variable $\lambda_{i}$ around the $k$-th saddle point has the scaling as $c_{k} / c_{k-1}$. Suppose we rescale the integration variables $\lambda_{i}^{L}$ using the largest scale $c_{1} / c_{0}$ and $\lambda_{i}^{R}$ using the smallest scale $c_{0} / c_{-1}$. Then the ratio $\lambda^{L} / \lambda^{R}$ will be the order of $\eta_{0}=\left(c_{1} c_{-1}\right) / c_{0}^{2} \ll 1$. Therefore, the dominant contribution of the determinant is

$$
\prod_{i=1}^{N_{L}} \prod_{j=1}^{N_{R}}\left(\lambda_{j}^{R}-\lambda_{i}^{L}\right)^{2 \beta}=\prod_{j=1}^{N_{R}}\left(\lambda_{j}^{R}\right)^{2 \beta N_{L}}\left(1+\mathcal{O}\left(\eta_{0}\right)\right) .
$$

In addition, with the rescaling of $\lambda^{L}=\frac{c_{1}}{c_{0}} \xi^{L}$ the potential $V_{+}\left(\lambda^{L}\right)$ is put into the form $V_{+}\left(\xi^{L} c_{1} / c_{0}\right)=-\sum_{t=1}^{n}\left(\eta_{0} \xi^{L}\right)^{t}\left(\prod_{j=1}^{t-1} \eta_{-j}^{t-j}\right) / t$ which vanishes as $\eta_{0} \rightarrow 0$ and is neglected. On the other hand, $V_{-}\left(\xi^{L} c_{1} / c_{0}\right)=\sum_{s=1}^{m}\left(\prod_{a=0}^{s-1} \eta_{s-a}^{a}\right) /\left(s\left(\xi^{L}\right)^{s}\right)$ is finite as $\eta_{0} \rightarrow 0$. As the result, the partition function of $\lambda^{L}$ has the form

$$
Z_{L}=\int\left[\prod_{i=1}^{N_{L}} d \lambda_{i}^{L}\right] \Delta\left(\lambda^{L}\right)^{2 \beta} \exp \left[-\frac{\sqrt{\beta}}{g} \sum_{i=1}^{N_{L}} V_{L}\left(\lambda_{i}^{L}\right)\right]
$$

where $V_{L}(z)=-c_{0} \log z+V_{-}(z)$. Therefore, $Z_{L}$ is identified as the inner product $\left\langle\Delta\left(c_{\infty}+\right.\right.$ $\left.\hbar b N_{R}\right)\left|I^{(m)}\right\rangle$ where the Liouville momentum at infinity is $c_{\infty}+\hbar b N_{R}$.

Likewise, $\lambda^{R}$ contribution is given by

$$
Z_{R}=\int\left[\prod_{j=1}^{N_{R}} d \lambda_{j}^{R}\right] \Delta\left(\lambda^{R}\right)^{2 \beta} \exp \left[-\frac{\sqrt{\beta}}{g} \sum_{j=1}^{N_{R}} V_{R}\left(\lambda_{j}^{R}\right)\right]
$$

where $V_{R}(z)=-\left(c_{0}+\hbar b N_{L}\right) \log z+V_{+}(z)$. Here, the potential $V_{R}(z)$ is not $-c_{0} \log z+V_{+}(z)$ but an extra term $-\hbar b N_{L} \log z$ is added due to the extra contribution of the Vandermode determinant (3.2). It is a simple exercise to show that $V_{-}\left(\xi^{R} c_{0} / c_{-1}\right) \rightarrow 0$ as $\eta_{0} \rightarrow 0$. After this consideration, one notices that $Z_{R}$ is identified with $\left\langle I^{(n)} \mid \Delta\left(c_{0}+\hbar b N_{L}\right)\right\rangle$.

Finally, the sub-dominant part of the determinant in (3.2) has the leading term

$$
-2 \beta Z_{L} Z_{R}\left[\sum_{i=1}^{N_{L}} \sum_{j=1}^{N_{R}}\left\langle\lambda_{i}^{L}\right\rangle_{L}\left\langle\frac{1}{\lambda_{j}^{R}}\right\rangle_{R}\right]
$$


$Z_{N}^{(2 ; 2)}$

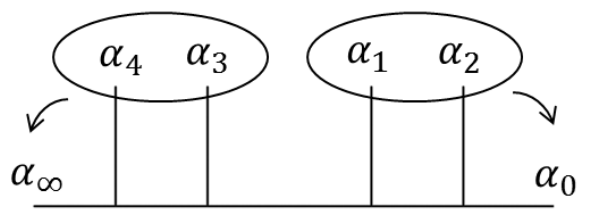

$Z_{L}$

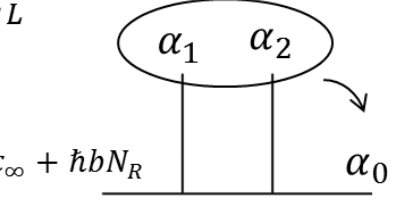

$\times$

$Z_{R}$

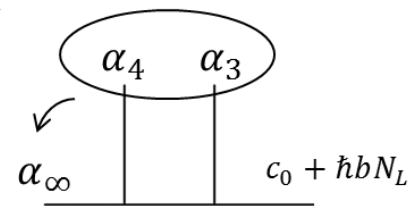

Figure 7. The case $m=n=2$. To the zeroth order of $\eta_{0}$, the partition function is factorized.

where $\langle\cdots\rangle_{L}$ and $\langle\cdots\rangle_{R}$ refer to the expectation value

$$
\langle\mathcal{O}\rangle_{A}=\frac{1}{Z_{A}} \int\left[\prod_{i=1}^{N_{A}} d \lambda_{i}^{A}\right] \Delta\left(\lambda^{A}\right)^{2 \beta} \mathcal{O} e^{-\frac{\sqrt{\beta}}{g} \sum_{i=1}^{N_{A}} V_{A}\left(\lambda_{i}^{A}\right)}, \quad A=L, R .
$$

Since the expectation value $\left\langle\lambda_{i}^{L}\right\rangle_{L}$ is $\mathcal{O}\left(c_{1} / c_{0}\right)$ and $\left\langle 1 / \lambda_{j}^{R}\right\rangle_{R} \sim \mathcal{O}\left(c_{-1} / c_{0}\right)$, its product is the order of $\eta_{0}$ and vanishes as $\eta_{0} \rightarrow 0$. The next leading contributions should vanish as the high power of $\eta_{0}$ 's.

Thus, one can conclude that $Z_{N}^{(n ; m)}=Z_{L} Z_{R}\left(1+\mathcal{O}\left(\eta_{0}\right)\right)$ and

$$
\left\langle I^{(n)} \mid I^{(m)}\right\rangle=\left\langle I^{(n)} \mid \Delta\left(c_{0}+\hbar b N_{L}\right)\right\rangle\left\langle\Delta\left(c_{\infty}+\hbar b N_{R}\right) \mid I^{(m)}\right\rangle\left(1+\mathcal{O}\left(\eta_{0}\right)\right) .
$$

This conclusion is checked by the explicit result given in the previous section. Since $\left\langle\Delta \mid I^{(2)}\right\rangle=\left\langle I^{(2)} \mid \Delta\right\rangle$ in $(2.20)$, we have the inner product $Z_{L}=\left\langle\Delta\left(c_{\infty}+\hbar b N_{R}\right) \mid I^{(m)}\right\rangle$

$$
-\hbar^{2} \log Z_{L}=\hbar b N_{L}\left(\hbar b N_{L}+2 c_{0}\right) \log c_{1}+\frac{\hbar b N_{2} c_{0}}{\eta_{1}}+\frac{\hbar b N_{2}}{2}\left(3 \hbar b N_{2}+4 c_{0}\right) \log \eta_{1}+\mathcal{O}\left(\eta_{1}\right)
$$

where $N_{L}=N_{1}+N_{2}$ and $N_{R}=N_{0}+N_{-1}$. And the inner product $Z_{R}=\left\langle I^{(2)} \mid \Delta\left(c_{0}+\hbar b N_{L}\right)\right\rangle$ is given as

$$
\begin{aligned}
-\hbar^{2} \log Z_{R}= & -\hbar b N_{R}\left(\hbar b N_{R}+2\left(c_{0}+\hbar b N_{L}\right)\right) \log c_{-1}-\frac{\hbar b N_{-1} c_{0}}{\eta_{-1}} \\
& -\frac{\hbar b N_{-1}}{2}\left(-3 \hbar b N_{-1}+4\left(c_{0}+\hbar b N\right)\right) \log \eta_{-1}+\mathcal{O}\left(\eta_{-1}\right)
\end{aligned}
$$

where the neutrality condition $c_{\infty}=-c_{0}-\hbar b N$ is used. Therefore, the product of $Z_{L}$ and $Z_{R}$ is given as

$$
\begin{aligned}
-\hbar^{2} \log \left(Z_{L} Z_{R}\right)= & -\hbar b N\left(\hbar b N+2 c_{0}\right) \log c_{-1}-\frac{\hbar b c_{0} N_{-1}}{\eta_{-1}}+\frac{\hbar b c_{0} N_{2}}{\eta_{1}} \\
& +\hbar b N_{L}\left(\hbar b N_{L}+2 c_{0}\right) \log \eta_{0}+\frac{\hbar b N_{2}\left(3 \hbar b N_{2}+4 c_{0}\right)}{2} \log \eta_{1} \\
& -\frac{\hbar b N_{-1}\left(-3 \hbar b N_{-1}+4\left(c_{0}+\hbar b N\right)\right)}{2} \log \eta_{-1}+\mathcal{O}\left(\eta_{-1}, \eta_{0}, \eta_{1}\right)
\end{aligned}
$$


where $N=N_{L}+N_{R}$. This result $Z_{L} Z_{R}$ is in perfect agreement with the inner product $\left\langle I^{(2)} \mid I^{(2)}\right\rangle$ shown in (2.57).

\subsection{Hierarchical relation between $\left\langle\Delta \mid I^{(m)}\right\rangle$ and $\left\langle\Delta \mid I^{(m-1)}\right\rangle$}

The singular contribution of $\left\langle I^{(n)} \mid I^{(m)}\right\rangle$ at small $\eta_{0}$ limit is given as the product of $\left\langle I^{(n)} \mid \Delta\right\rangle$ and $\left\langle\Delta \mid I^{(m)}\right\rangle$. What will happen to the other parameters?

Let us consider $\left\langle\Delta \mid I^{(m)}\right\rangle$. The potential has $m$ saddle points, $V(z)=-c_{0} \log z+V_{-}(z)$ in (2.36). Let us concentrate on the integration variable $\lambda_{i}^{(m)}$ around the $m$-th saddle point whose index $i$ running from 1 to $N_{m}$. The other variables $\lambda_{J}$ has the index $J$ running from 1 to $\bar{N}_{m}$ so that $\bar{N}_{m}+N_{m}=N$.

The $m$-th saddle point is much smaller than other saddle points $\lambda_{i}^{(m)} \ll \lambda_{J}$ and therefore, $\eta_{m-1} \ll 1$. The determinant part $\prod_{J=1}^{\bar{N}_{m}} \prod_{i=1}^{N_{m}}\left(\lambda_{J}-\lambda_{i}^{(m)}\right)^{2 \beta}$ has the dominant contribution $\prod_{J=1}^{\bar{N}_{m}}\left(\lambda_{J}\right)^{2 \beta N_{m}}$. The partition function with $\lambda_{J}$ 's only is $Z^{(m-1)}$ :

$$
Z^{(m-1)} \equiv \int\left[\prod_{J} d \lambda_{J}\right] \Delta\left(\lambda_{J}\right)^{2 \beta} \exp \left[-\frac{\sqrt{\beta}}{g} \sum_{J} V_{m-1}\left(\lambda_{J}\right)\right] .
$$

The effective potential $V_{m-1}(z)$ contains $-\hbar b N_{m} \log z$ from the determinant part. In addition, as $\eta_{m-1} \rightarrow 0$, the original term $c_{m} / z^{m}$ in the potential $V(z)$ drops out. This is easily seen if one scales $\lambda_{J}$ by $c_{k} / c_{k-1}$ for any $k \leq m-1$. While $\xi_{J}=\mathcal{O}\left(\eta_{m-1}^{0}\right)$, the term $c_{m} / z^{m}$ is $\mathcal{O}\left(\eta_{m-1}\right)$. Other terms are finite. Therefore we have the effective potential

$$
V_{m-1}(z)=-\left(c_{0}+\hbar b N_{m}\right) \log z+\sum_{s=1}^{m-1} \frac{c_{s}}{s z^{s}}
$$

The partition function $Z^{(m-1)}$ is the inner product $\left\langle\Delta \mid I^{(m-1)}\left(c_{0}+\hbar b N_{m}\right)\right\rangle$ and the irregular vector $\left|I^{(m-1)}\left(c_{0}+\hbar b N_{m}\right)\right\rangle$ has the rank $m-1$ with the Liouville momentum $\alpha=c_{0}+\hbar b N_{m}$.

The partition function written in terms of $\lambda_{i}^{(m)}$ has the form

$$
T^{(m)} \equiv \int\left[\prod_{i} d \lambda_{i}^{(m)}\right] \Delta\left(\lambda^{(m)}\right)^{2 \beta} \exp \left[-\frac{\sqrt{\beta}}{g} \sum_{i} V\left(\lambda_{i}^{(m)}\right)\right] .
$$

Here, the original potential $V(z)$ is used and becomes infinite as $\eta_{m-1} \rightarrow 0$. This shows that $T^{(m)}$ contains the singular contribution.

It is noted that the sub-leading contribution in the determinant vanishes as $\eta_{m-1} \rightarrow 0$. To check this, let us consider the $\mathcal{O}\left(\lambda_{i}^{(m)} / \lambda_{J}\right)$ contribution

$$
Z^{(m-1)} T^{(m)}\left[\sum_{i=1}^{N_{m}} \sum_{J=1}^{\bar{N}_{m}}\left\langle\lambda_{i}^{(m)}\right\rangle_{T^{(m)}}\left\langle\frac{1}{\lambda_{J}}\right\rangle_{Z^{(m-1)}}\right]
$$

The expectation value $\left\langle\lambda_{i}^{(m)}\right\rangle_{T^{(m)}}$ is $\mathcal{O}\left(c_{m-2} / c_{m-1}\right)$ and $\left\langle 1 / \lambda_{J}\right\rangle_{Z^{(m-1)}}$ is $\mathcal{O}\left(c_{m} / c_{m-1}\right)$. Therefore, its product is the order of $\eta_{m-1}$. The higher order contribution is given as the higher power of $\eta_{m-1}$ 's. 


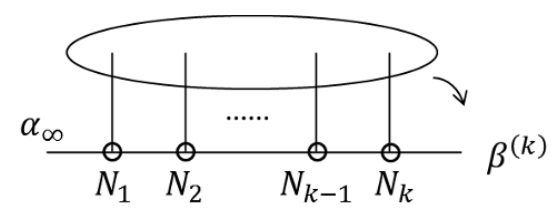

$$
\text { I } \lim _{x \rightarrow 0}
$$

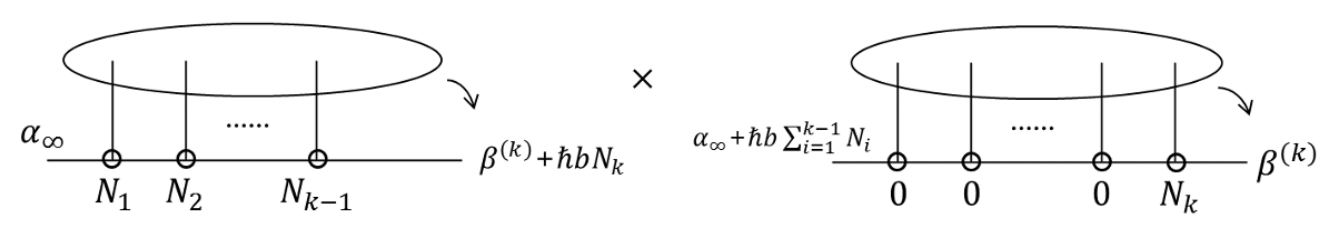

Figure 8. Hierarchical structure of $\left\langle\Delta \mid I^{(k)}\left(\beta^{(k)}\right)\right\rangle$ as $\eta_{k-1} \rightarrow 0$.

Considering all the contributions, one concludes that $\left\langle\Delta \mid I^{(m)}\right\rangle=Z^{(m-1)} T^{(m)} \times$ $\left(1+\mathcal{O}\left(\eta_{m-1}\right)\right)$ which shows that

$$
\left\langle\Delta \mid I^{(m)}\left(c_{0}\right)\right\rangle=T^{(m)}\left(c_{0} ; N_{m}\right)\left\langle\Delta \mid I^{(m-1)}\left(c_{0}+\hbar b N_{m}\right)\right\rangle\left(1+\mathcal{O}\left(\eta_{m-1}\right)\right) .
$$

In the small $\eta_{m-1}$ limit, the irregular vector of rank $m$ is reduced to the one of the rank $m-1$ with the momentum shift $c_{0} \rightarrow c_{0}+\hbar b N_{m}$ and its non-trivial ratio $T^{(m)}\left(c_{0} ; N_{m}\right)$ contains all the singular contribution. When $m=1$, one has the singular structure of $\left\langle\Delta \mid I^{(1)}\left(c_{0}\right)\right\rangle$ and $T^{(1)}\left(c_{0} ; N_{1}\right)$ is the same.

One may apply this result (3.16) successively to get the singular part of $\left\langle\Delta \mid I^{(m)}\left(c_{0}\right)\right\rangle$ when the set of parameters $\left\{\eta_{1}, \cdots, \eta_{m-1}\right\}$ are small. As the result we have the following hierarchical structure of the singularity.

$$
\left\langle\Delta \mid I^{(m)}\left(c_{0}\right)\right\rangle_{S}=\prod_{k=1}^{m} T^{(k)}\left(\beta^{(k)} ; N_{k}\right)
$$

where the subscript $S$ stands for the singular part only neglecting regular contributions. Here $T^{(k)}\left(\beta^{(k)} ; N_{k}\right)$ is defined in (3.14) which has filling fraction $N_{k}$ and Liouiville momen$\operatorname{tum} \beta^{(k)}$ satisfies the relation $\beta^{(k-1)}=\beta^{(k)}+\hbar b N_{k}$ and $\beta^{(m)}=c_{0}$.

The explicit expression of $T^{(k)}\left(\beta ; N_{k}\right)$ can be found from the expressions in section 2 . For example, using the inner product $\left\langle\Delta \mid I^{(2)}\left(c_{0}\right)\right\rangle$ given in $(2.20)$, one has $T^{(2)}\left(c_{0} ; N_{2}\right)$ by putting $N_{1} \rightarrow 0$

$$
T^{(2)}\left(c_{0} ; N_{2}\right)=\left(c_{1}\right)^{-b N_{2}\left(b N_{2}+2 \frac{c_{0}}{\hbar}\right)}\left(\eta_{1}\right)^{-\frac{b N_{2}}{2}\left(3 b N_{2}+4 \frac{c_{0}}{\hbar}\right)} e^{-\frac{b N_{2} c_{0} / \hbar}{\eta_{1}}+\mathcal{O}\left(\eta_{1}\right)} .
$$

When $m=3,\left\langle\Delta \mid I^{(3)}\left(c_{0}\right)\right\rangle$ in $(2.35)$ reduces to $T^{(3)}\left(c_{0} ; N_{3}\right)$ if $N_{1}=N_{2} \rightarrow 0$

$$
\begin{aligned}
& T^{(3)}\left(c_{0} ; N_{3}\right)=\left(c_{1}\right)^{-b N_{3}\left(b N_{3}+2 \frac{c_{0}}{\hbar}\right)}\left(\eta_{1}\right)^{-\frac{b N_{3}}{2}\left(3 b N_{3}+4 \frac{c_{0}}{\hbar}\right)} \\
&\left(\eta_{2}\right)^{-2 b N_{3}\left(b N_{3}+\frac{c_{0}}{\hbar}\right)} e^{-\frac{b c_{0}}{\eta_{1} \hbar}\left(-N_{3}+\frac{2 N_{3}}{\eta_{2}}-\frac{N_{3}}{3 \eta_{2}^{2}}\right)+\mathcal{O}\left(\eta_{1}, \eta_{2}\right)} .
\end{aligned}
$$


We note that the singular structure in (3.17) is consistent with the ansatz proposed in [6]. When $k=2$, the ansatz is given as

$$
\left|I^{(2)}\left(\beta^{(2)}\right)\right\rangle=c_{2}^{\nu_{2}} c_{1}^{\nu_{1}} e^{\left(\beta^{(2)}-\beta^{(1)}\right) \frac{c_{1}^{2}}{c_{2}}} \sum_{j=0}^{\infty} c_{2}^{j}\left|I_{2 j}^{(1)}\left(\beta^{(1)}\right)\right\rangle
$$

where the vector $\left|I_{2 j}^{(1)}\left(\beta^{(1)}\right)\right\rangle$ is so-called generalized descendants of the rank 1 irregular vector and is the linear combination of vectors obtained by acting Virasoro generators or $c_{1}$-derivatives on $\left|I_{0}^{(1)}\left(\beta^{(1)}\right)\right\rangle \equiv\left|I^{(1)}\left(\beta^{(1)}\right)\right\rangle$. From the Ward identities the factors were determined as $\nu_{1}=2\left(\beta^{(2)}-\beta^{(1)}\right)\left(Q-\beta^{(1)}\right), \nu_{2}=\left(\beta^{(1)}-\beta^{(2)}\right)\left(\frac{3}{2} Q-\frac{3}{2} \beta^{(1)}-\frac{1}{2} \beta^{(2)}\right)$. The prefactor in front of $\left|I_{0}^{(1)}\left(\beta^{(1)}\right)\right\rangle$ is $T^{(2)}\left(\beta^{(2)} ; N_{2}\right) .(Q=0$ if the large $N$ limit is taken.)

\subsection{Flow equations for $T^{(k)}\left(\beta^{(k)} ; N_{k}\right)$}

It is shown that the singular structure of the inner product between the irregular vectors are encoded in the partition function $T^{(k)}$ as in (3.17). Therefore, if we find $T^{(k)}$, then all the singular structures in the parameter space are known. In this subsection, we are presenting a set of differential equations for $T^{(k)}$ so that one can find the partition function directly using the flow equations.

We start with the partition function

$$
T^{(k)}\left(\beta^{(k)} ; N_{k}\right)=\int\left[\prod_{i=1}^{N_{k}} d \lambda_{i}\right] \Delta(\lambda)^{2 \beta} \exp \left[-\frac{\sqrt{\beta}}{g} \sum_{i=1}^{N_{k}} V\left(\lambda_{i}\right)\right]
$$

where we use the potential $V(z)=-\beta^{(k)} \log z+\sum_{t=1}^{k} c_{t} /\left(t z^{t}\right)$. If one scale $\lambda_{i}^{(k)}$ by $c_{k} / c_{k-1}$, one has $T^{(k)}\left(\beta^{(k)} ; N_{k}\right)=\left(c_{k} / c_{k-1}\right)^{N_{k}-b^{2} N_{k}\left(N_{k}-1\right)-2 \beta^{(k)} b N_{k} / \hbar} \tilde{T}^{(k)}$ where $\tilde{T}^{(k)}$ is given in terms of the rescaled integration variables $\xi_{i}$. Its potential is given as

$$
\tilde{V}(\xi)=\frac{\beta^{(k)}}{\kappa_{0}}\left(-\kappa_{0} \log \xi+\sum_{t=1}^{k} \frac{\kappa_{t}}{t \xi^{t}}\right) \text {. }
$$

The parameters $\left\{\eta_{1}, \cdots, \eta_{k-1}\right\}$ are replaced with $\kappa_{t} \equiv \prod_{\ell=1}^{k-1-t} \eta_{t+\ell}^{\ell}$ for $(0 \leq t \leq k-2)$ and $\kappa_{k-1}=\kappa_{k} \equiv 1$. The overall parameter $\kappa_{0}$ is specially treated and is equivalently called $\tau_{k}$. These new parameters have a definite ordering: $\tau_{k} \ll \kappa_{1} \ll \cdots \ll \kappa_{k-2} \ll 1$. In this rescaling, $\xi_{i}$ around the $k$-th saddle point and has $\left\langle\xi_{i}^{s}\right\rangle=(-1)^{s}+\mathcal{O}\left(\left\{\kappa_{\ell}\right\}\right)$.

Defining $\tilde{H}^{(k)}\left(\left\{\kappa_{\ell}\right\}\right) \equiv-\hbar^{2} \log \tilde{T}^{(k)}$, one has $(k-1)$-set of differential equations

$$
\begin{aligned}
\frac{\partial \tilde{H}^{(k)}}{\partial \tau_{k}} & =\frac{2 \hbar b \beta^{(k)}}{\tau_{k}^{2}} \sum_{t=1}^{k} \frac{\kappa_{t}}{t}\left\langle\sum_{i=1}^{N_{k}} \frac{1}{\xi_{i}{ }^{t}}\right\rangle \\
\frac{\partial \tilde{H}^{(k)}}{\partial \kappa_{t}} & =-\frac{2 \hbar b \beta^{(k)}}{t \tau_{k}}\left\langle\sum_{i=1}^{N_{k}} \frac{1}{\xi_{i}{ }^{t}}\right\rangle, \quad(1 \leq t \leq k-2)
\end{aligned}
$$

Since $\left\langle 1 / \xi_{i}^{t}\right\rangle=\mathcal{O}\left(\left\{\tau_{k}^{0}\right\}\right)$, we may put the flow equation in power series of $\tau_{k}$ as

$$
\frac{\partial \tilde{H}^{(k)}}{\partial \tau_{k}}=-\frac{H_{-1}^{(k)}}{\tau_{k}^{2}}+\frac{H_{0}^{(k)}}{\tau_{k}}+\sum_{n \geq 1} n H_{n}^{(k)} \tau_{k}^{n-1}, \quad \frac{\partial \tilde{H}^{(k)}}{\partial \kappa_{t}}=\sum_{n \geq-1} G_{t, n}^{(k)} \tau_{k}^{n}
$$


where $H_{n}^{(k)}$ and $G_{t, n}^{(k)}$ are independent of $\tau_{k}$ and regular in $\left\{\kappa_{1}, \cdots, \kappa_{k-2}\right\}$. Therefore, if one integrates (3.25) over $\tau_{k}$ one ends up with the form

$$
\tilde{H}^{(k)}\left(\left\{\kappa_{\ell}\right\}\right)=\frac{H_{-1}^{(k)}}{\tau_{k}}+H_{0}^{(k)} \log \tau_{k}+\sum_{n \geq 1} H_{n}^{(k)} \tau_{k}^{n} .
$$

This shows that the singular contribution to $T^{(k)}$ as $\eta_{\ell} \rightarrow 0$ is due to the terms $H_{-1}^{(k)}, H_{0}^{(k)}$.

\subsection{Evaluation of $T^{(k)}$ from the flow equation}

The direct calculation of the partition function uses the loop equation and requires the complicated integration to find the filling fraction (2.15). This is not always the case. However, it can be demonstrated that $T^{(k)}\left(\beta^{(k)} ; N_{k}\right)$ does not need any explicit integration of the filling fraction. This is done by fully exploiting the flow equation.

The starting point is to observe that the flow equation (3.25) must satisfy the selfconsistency condition

$$
\frac{\partial^{2} \tilde{H}^{(k)}}{\partial \kappa_{a} \partial \kappa_{b}}=\frac{\partial^{2} \tilde{H}^{(k)}}{\partial \kappa_{b} \partial \kappa_{a}} \quad(0 \leq a, b \leq k-2)
$$

This trivially looking conditions provide a very powerful tool to find $H_{n}^{(k)}$. For example, the self-consistency shows that $H_{0}^{(k)}$ is a constant and is independent of any $\kappa_{\ell}$. Not only that, the consistency condition turns out to constrain all the expectation values $\left\langle\sum_{i} 1 / \xi_{i}^{t}\right\rangle$ needed for $H_{-1}^{(k)}$.

Let us redefine the expectation value using a new parameter

$$
\tilde{D}_{s} \equiv-2 \hbar b \beta^{(k)} \sum_{t=s+1}^{k} \kappa_{t}\left\langle\sum_{i=1}^{N_{k}} \frac{1}{\xi_{i}^{t-s}}\right\rangle
$$

and put the flow equation in terms of $\tilde{D}_{s}$

$$
\begin{aligned}
\frac{\partial \tilde{H}^{(k)}}{\partial \tau_{k}} & =-\frac{1}{\tau_{k}^{2}} \sum_{s=0}^{k-1} \tilde{D}_{s}\left(\sum_{j=k-s}^{k} \frac{\kappa_{j}}{j} A_{j-(k-s)}\right) \\
\frac{\partial \tilde{H}^{(k)}}{\partial \kappa_{t}} & =\frac{1}{t \tau_{k}} \sum_{s=k-t}^{k-1} \tilde{D}_{s} A_{s-(k-t)}
\end{aligned}
$$

where $A_{\ell}=-\left(A_{\ell-1}+\kappa_{k-2} A_{\ell-2}+\cdots+\kappa_{k-\ell} A_{0}\right)$ and $A_{0}=1$.

To make the consistency condition more tractable, we expand $\tilde{D}_{s}$ in power series of $\left\{\kappa_{\ell}\right\}$ since $\left\langle 1 / \xi_{i}^{t}\right\rangle=\mathcal{O}\left(\left\{\kappa_{k}^{0}\right\}\right)$.

$$
\tilde{D}_{s}=\sum_{\left\{\alpha_{\ell} \geq 0\right\}}\left[\tilde{D}_{s}\right]_{\alpha_{0}, \alpha_{1}, \cdots, \alpha_{k-2}} \tau_{k}^{\alpha_{0}} \kappa_{1}^{\alpha_{1}} \cdots \kappa_{k-2}^{\alpha_{k-2}} .
$$

The power expansion allows one to identify $H_{\ell}^{(k)}$ explicitly. Comparing (3.25) with (3.29) and (3.30) one has $H_{-1}^{(k)}$ using the zeroth order of $\tau_{k}$ in $\tilde{D}_{s}$.

$$
H_{-1}^{(k)}=\sum_{s=0}^{k-1} \sum_{j=k-s}^{k} \sum_{\left\{\alpha_{\ell} \geq 0\right\}} \frac{\kappa_{j}}{j} A_{j-(k-s)}\left[\tilde{D}_{s}\right]_{0, \alpha_{1}, \cdots, \alpha_{k-2}} \kappa_{1}^{\alpha_{1}} \cdots \kappa_{k-2}^{\alpha_{k-2}} .
$$


Let us find out how useful the flow equation is. When $k=2$, we have only one flow equation with $\tau_{2}=\eta_{1}$.

$$
\frac{\partial \tilde{H}^{(2)}}{\partial \tau_{2}}=-\frac{\tilde{D}_{0}+\tilde{D}_{1}}{2 \tau_{2}^{2}} .
$$

Once $\tau_{2}$ dependence of $\left(\tilde{D}_{0}+\tilde{D}_{1}\right)$ is known, one find $\tilde{H}^{(2)}$ completely. For this, the loop equation provides a useful information. The loop equation for $T^{(k)}$ is given as.

$$
\tilde{f}(z)=4 \tilde{W}(z)^{2}-4 \tilde{V}^{\prime}(z) \tilde{W}(z)+2 \hbar Q \tilde{W}^{\prime}(z)-\hbar^{2} \tilde{W}(z, z)
$$

where $2 \tilde{W}(z)=\hbar b\left\langle\sum_{i=1}^{N_{k}} 1 /\left(z-\xi_{i}\right)\right\rangle$ and $\tilde{W}(z, w)=-b^{2}\left\langle\sum_{i, j=1}^{N_{k}} \frac{1}{\left(z-\xi_{i}\right)\left(w-\xi_{j}\right)}\right\rangle_{c}$ is the connected 2-point resolvent. Then the quantum correction $\tilde{f}(z)$ contains $\tilde{D}_{s}$ 's

$$
\tilde{f}(z)=2 \hbar b\left\langle\sum_{i=1}^{N_{k}} \frac{-\tilde{V}^{\prime}(z)+\tilde{V}^{\prime}\left(\xi_{i}\right)}{z-\xi_{i}}\right\rangle=\frac{1}{\tau_{k}} \sum_{s=0}^{k-1} \frac{\tilde{D}_{s}}{z^{2+s}} .
$$

One can collect the data of $\tilde{D}_{s}$ after the large $z$ expansion of (3.34)

$$
\begin{aligned}
& {\left[\tilde{D}_{0}\right]_{0}=0 \quad\left[\tilde{D}_{0}\right]_{1}=\hbar b N_{2}\left(\hbar b N_{2}+2 \beta^{(2)}-\hbar Q\right)} \\
& {\left[\tilde{D}_{1}\right]_{0}=2 \hbar b N_{2} \beta^{(2)}, \quad\left[\tilde{D}_{1}\right]_{1}=-2 \hbar b N_{2}\left(\hbar b N_{2}+\beta^{(2)}-\hbar Q\right) .}
\end{aligned}
$$

This simple data is enough to obtain the singular part

$$
\tilde{H}^{(2)}=\frac{\hbar b N_{2} \beta^{(2)}}{\tau_{2}}+\frac{\hbar b N_{2}}{2}\left(\hbar b N_{2}-\hbar Q\right) \log \tau_{2}+\mathcal{O}\left(\tau_{2}\right)
$$

and thus, we have

$$
T^{(2)}\left(\beta^{(2)} ; N_{2}\right)=\left(\frac{c_{2}}{c_{1}}\right)^{-b N_{2}\left(b N_{2}+2 \beta^{(2)} / \hbar-Q\right)}\left(\tau_{2}\right)^{-\frac{b N_{2}\left(b N_{2}-Q\right)}{2}} e^{-\frac{b N_{2} \beta^{(2)}}{\tau_{2} \hbar}+\mathcal{O}\left(\tau_{2}\right)}
$$

If one puts $\beta^{(2)}=c_{0}$ and $Q=0$, we reproduce the result in (3.18). This example shows that one may obtain the singular contribution of $T^{(k)}$ directly. We do not need any explicit integration of the filling fraction as in section 2 to connect the partition function with the filling fraction. Here, the flow equations together with the simple analytic structure of the loop equation is enough to find all the singular structures of the inner product.

When $k=3$, there are two flow equations. Using the parameters $\tau_{3}=\eta_{1} \eta_{2}^{2}$ and $\kappa_{1}=\eta_{2}$, we have the equations

$$
\begin{aligned}
\frac{\partial \tilde{H}^{(3)}}{\partial \tau_{3}} & =-\frac{\left(4 \kappa_{1}-1\right) \tilde{D}_{2}+\tilde{D}_{1}+2 \tilde{D}_{0}}{6 \tau_{3}^{2}} \\
\frac{\partial \tilde{H}^{(3)}}{\partial \kappa_{1}} & =\frac{\tilde{D}_{2}^{(3)}}{\tau_{3}}
\end{aligned}
$$

The self-consistency $\partial^{2} \tilde{H}^{(3)} / \partial \tau_{3} \partial \kappa_{1}=\partial^{2} \tilde{H}^{(3)} / \partial \kappa_{1} \partial \tau_{3}$ gives the recursion relation:

$$
(m+1)\left(\left[\tilde{D}_{2}\right]_{n, m+1}-\left[\tilde{D}_{1}\right]_{n, m+1}\right)=2(3 n+2 m-1)\left[\tilde{D}_{2}\right]_{n, m} .
$$


To solve the recursion relations we need the initial conditions such as $\left[\tilde{D}_{1}\right]_{n, \alpha_{1}}$ for $\alpha_{1} \geq 1$ and $\left[\tilde{D}_{2}\right]_{n, 0}$. The initial conditions are provided by the loop equation (3.34). Note that $\tilde{D}_{s}$ is given for arbitrary $k$

$$
\begin{aligned}
\tilde{D}_{s}= & \hbar^{2} b^{2} \tau_{k} \sum_{t=0}^{s}\left\langle\sum_{i=1}^{N_{k}} \xi_{i}{ }^{t}\right\rangle\left\langle\sum_{i=1}^{N_{k}} \xi_{i}{ }^{s-t}\right\rangle+2 \hbar b \beta^{(k)} \sum_{t=0}^{s} \kappa_{t}\left\langle\sum_{i=1}^{N_{k}} \xi_{i}{ }^{s-t}\right\rangle \\
& -(s+1) \hbar^{2} b Q \tau_{k}\left\langle\sum_{i=1}^{N_{k}} \xi_{i}{ }^{s}\right\rangle+\hbar^{2} b^{2} \tau_{k} \sum_{t+m=s}\left\langle\sum_{i=1}^{N_{k}} \xi_{i}^{t} \sum_{j=1}^{N_{k}} \xi_{j}^{m}\right\rangle .
\end{aligned}
$$

Using the fact $\left\langle\sum_{i=1}^{N_{k}} \xi_{i}\right\rangle=-N_{k}+\mathcal{O}\left(\kappa_{\ell}\right)$, we have at the zero-th order of $\tau_{k}$

$$
\begin{aligned}
& {\left[\tilde{D}_{s}\right]_{0, \cdots, 0, \alpha_{t}=1,0, \cdots, 0}=(-1)^{s-t} 2 \hbar b N_{k} \beta^{(k)} \quad(1 \leq t \leq s \leq k-2)} \\
& {\left[\tilde{D}_{k-1}\right]_{0, \cdots, 0}=2 \hbar b N_{k} \beta^{(k)}} \\
& {\left[\tilde{D}_{k-1}\right]_{0, \cdots, 0, \alpha_{t}=1,0, \cdots, 0}=(-1)^{k-1-t} 2 \hbar b N_{k} \beta^{(k)} \quad(1 \leq t \leq k-2) .}
\end{aligned}
$$

In addition, unless $\alpha_{s}=1$ and $\alpha_{s+1}=\cdots=\alpha_{k-2}=0$ we have

$$
\left[\tilde{D}_{s}\right]_{0, \cdots, 0, \alpha_{s}, \alpha_{s+1}, \cdots, \alpha_{k-2}} \equiv 0 \quad \text { for } s \leq k-2 .
$$

At the first order of $\tau_{k}$, we have

$$
\left[\tilde{D}_{s}\right]_{1,0, \cdots, 0}=(-1)^{s} \hbar b N_{k}\left((s+1) \hbar b N_{k}+2 \beta^{(k)}-(s+1) \hbar Q\right) .
$$

Since $H_{0}^{(k)}$ is a constant, independent of $\kappa_{\ell}$ 's, one can find $H_{0}^{(k)}$ using the flow equation (3.29). From the first order of $\tau_{k}$ in $\tilde{D}_{s}$, one has

$$
H_{0}^{(k)}=-\sum_{s=0}^{k-1} \sum_{j=k-s}^{k} \sum_{\left\{\alpha_{\ell} \geq 0\right\}} \frac{\kappa_{j}}{j} A_{j-(k-s)}\left[\tilde{D}_{s}\right]_{1, \alpha_{1}, \cdots, \alpha_{k-2}} \kappa_{1}^{\alpha_{1}} \cdots \kappa_{k-2}^{\alpha_{k-2}} .
$$

All the terms which depend on $\kappa_{\ell}$ should be cancelled. Noting that $\kappa_{k-1}=\kappa_{k}=1$ and $A_{\ell}=(-1)^{\ell}+\mathcal{O}\left(\left\{\kappa_{\ell}\right\}\right)$ and changing the order of the sum over $s$ and $j$, one obtains

$$
H_{0}^{(k)}=-\sum_{j=0}^{1} \sum_{\ell=0}^{k-1-j} \frac{(-1)^{\ell}}{k-j}\left[\tilde{D}_{j+\ell}\right]_{1,0, \cdots, 0}=\frac{\hbar b N_{k}}{2}\left(\hbar b N_{k}-\hbar Q\right) .
$$

Now back to the case $k=3$, we have data from the loop equation such as $\left[\tilde{D}_{1}\right]_{0, m}=$ $-2 \hbar b N_{3} \beta^{(3)} \delta_{m, 1}$ and $\left[\tilde{D}_{2}\right]_{0,0}=2 \hbar b N_{3} \beta^{(3)}$. Solving the recursion relation (3.40), one has $\left[\tilde{D}_{2}\right]_{0,1}=-2 \hbar b N_{3} \beta^{(3)}$ which is consistent with the data (3.44) already obtained from the loop equation. Furthermore, $\left[\tilde{D}_{2}\right]_{0,2}=-2 \hbar b N_{3} \beta^{(3)}$. Finally the flow equation shows that

$$
\tilde{H}^{(3)}=-\frac{\hbar b \beta^{(3)} N_{3}}{3 \tau_{3}}+2 \hbar b \beta^{(3)} N_{3} \frac{\kappa_{1}}{\tau_{3}}-\hbar b \beta^{(3)} N_{3} \frac{\kappa_{1}^{2}}{\tau_{3}}+\frac{\hbar b N_{3}}{2}\left(\hbar b N_{3}-\hbar Q\right) \log \tau_{3}+\mathcal{O}\left(\tau_{3}\right)
$$

and therefore,

$$
T^{(3)}\left(\beta^{(3)} ; N_{3}\right)=\left(\frac{c_{3}}{c_{2}}\right)^{-b N_{3}\left(b N_{3}+2 \frac{\beta^{(3)}}{\hbar}-Q\right)}\left(\tau_{3}\right)^{-\frac{b N_{3}\left(b N_{3}-Q\right)}{2}} e^{-\frac{b \beta^{(3)} N_{3}}{\tau_{3} \hbar}\left(-\kappa_{1}^{2}+2 \kappa_{1}-\frac{1}{3}\right)+\mathcal{O}\left(\tau_{3}\right)} .
$$


The singular structure is the same as the one in (3.19) where $\beta^{(3)}=c_{0}$ and $Q=0$ are used.

In general, one can prove that $T^{(k)}$ can be obtained from the consistency condition together with the loop equation for $k \geq 3$. The proof is presented in the appendix. To present this idea more concretely, we explicitly calculate the case $k=4$. The flow equations are given by

$$
\begin{aligned}
& \frac{\partial \tilde{H}^{(4)}}{\partial \tau_{4}}=-\frac{1}{12 \tau_{4}^{2}}\left[3 \tilde{D}_{0}+\tilde{D}_{1}+\left(3 \kappa_{2}-1\right) \tilde{D}_{2}+\left(-4 \kappa_{2}+9 \kappa_{1}+1\right) \tilde{D}_{3}\right] \\
& \frac{\partial \tilde{H}^{(4)}}{\partial \kappa_{1}}=\frac{\tilde{D}_{3}}{\tau_{4}}, \quad \frac{\partial \tilde{H}^{(4)}}{\partial \kappa_{2}}=\frac{\tilde{D}_{2}-\tilde{D}_{3}}{2 \tau_{4}} .
\end{aligned}
$$

where $\kappa_{1}=\eta_{2} \eta_{3}^{2}, \kappa_{2}=\eta_{3}$ and $\tau_{4}=\eta_{1} \eta_{2}^{2} \eta_{3}^{3}$. Using the consistency of the flow equations

$$
\frac{\partial^{2} \tilde{H}^{(4)}}{\partial \tau_{4} \partial \kappa_{2}}=\frac{\partial^{2} \tilde{H}^{(4)}}{\partial \kappa_{2} \partial \tau_{4}}, \quad \frac{\partial^{2} \tilde{H}^{(4)}}{\partial \kappa_{1} \partial \kappa_{2}}=\frac{\partial^{2} \tilde{H}^{(4)}}{\partial \kappa_{2} \partial \kappa_{1}}, \quad \frac{\partial^{2} \tilde{H}^{(4)}}{\partial \tau_{4} \partial \kappa_{1}}=\frac{\partial^{2} \tilde{H}^{(4)}}{\partial \kappa_{1} \partial \tau_{4}},
$$

we have the recursion relations:

$$
\begin{gathered}
2(m+1)\left[\tilde{D}_{3}\right]_{n, \ell, m+1}=(\ell+1)\left[\tilde{D}_{2}\right]_{n, \ell+1, m}-(\ell+1)\left[\tilde{D}_{3}\right]_{n, \ell+1, m} \\
(-12 n-9 \ell+3)\left[\tilde{D}_{3}\right]_{n, \ell, m}+4(\ell+1)\left[\tilde{D}_{3}\right]_{n, \ell+1, m-1}-(\ell+1)\left[\tilde{D}_{3}\right]_{n, \ell+1, m} \\
=(\ell+1)\left[\tilde{D}_{1}\right]_{n, \ell+1, m}+3(\ell+1)\left[\tilde{D}_{2}\right]_{n, \ell+1, m-1}-(\ell+1)\left[\tilde{D}_{2}\right]_{n, \ell+1, m} \\
(6 n+4 m-2)\left[\tilde{D}_{3}\right]_{n, \ell, m}-(m+1)\left[\tilde{D}_{3}\right]_{n, \ell, m+1}-9(m+1)\left[\tilde{D}_{3}\right]_{n, \ell-1, m+1} \\
=(m+1)\left[\tilde{D}_{1}\right]_{n, \ell, m+1}-(m+1)\left[\tilde{D}_{2}\right]_{n, \ell, m+1}+(6 n+3 m-3)\left[\tilde{D}_{2}\right]_{n, \ell, m}
\end{gathered}
$$

The coefficients needed for the singular part $H_{-1}^{(4)}$ are $\left[\tilde{D}_{s}\right]_{0,0, m}$ with $m=0,1,2,3$ and $\left[\tilde{D}_{s}\right]_{0,1, m}$ with $m=0,1$. The initial data are given in $(3.42)-(3.45) ;\left[\tilde{D}_{1}\right]_{0, \alpha_{1}, \alpha_{2}},\left[\tilde{D}_{2}\right]_{0,0, \alpha_{2}}$ for arbitrary $\alpha_{1}, \alpha_{2} \in \mathbb{Z}^{+}$and $\left[\tilde{D}_{2}\right]_{0,1,0},\left[\tilde{D}_{3}\right]_{0,0,0},\left[\tilde{D}_{3}\right]_{0,1,0},\left[\tilde{D}_{3}\right]_{0,0,1}$. The recursion relation are solved to have $\left[\tilde{D}_{2}\right]_{0,1,1}=-2 \hbar b N_{4} \beta^{(4)},\left[\tilde{D}_{3}\right]_{0,1,1}=6 \hbar b N_{4} \beta^{(4)},\left[\tilde{D}_{3}\right]_{0,0,2}=-2 \hbar b N_{4} \beta^{(4)}$, $\left[\tilde{D}_{3}\right]_{0,0,3}=-4 \hbar b N_{4} \beta^{(4)}$. Therefore,

$$
\begin{aligned}
& \tilde{H}^{(4)}=\frac{\hbar b N_{4} \beta^{(4)}}{\tau_{4}}\left(2 \kappa_{1}-2 \kappa_{1} \kappa_{2}-\kappa_{2}+\kappa_{2}^{2}+\frac{\kappa_{2}^{3}}{3}+\frac{1}{6}\right)+\frac{\hbar b N_{4}}{2}\left(\hbar b N_{4}-\hbar Q\right) \log \tau_{4}+\mathcal{O}\left(\tau_{4}\right) \\
& T^{(4)}\left(\beta^{(4)} ; N_{4}\right)=\left(\frac{c_{4}}{c_{3}}\right)^{-b N_{4}\left(b N_{4}+2 \beta^{(4)} / \hbar-Q\right)}\left(\tau_{4}\right)^{-\frac{b N_{4}\left(b N_{4}-Q\right)}{2}} \\
& e^{-\frac{b N_{4} \beta^{(4)}}{\hbar \tau_{4}}\left(2 \kappa_{1}-2 \kappa_{1} \kappa_{2}-\kappa_{2}+\kappa_{2}^{2}+\frac{\kappa_{2}^{3}}{3}+\frac{1}{6}\right)+\mathcal{O}\left(\tau_{4}\right)} .
\end{aligned}
$$

Using the hierarchical structure (3.17), we obtain the singular part of the partition function

$$
\begin{aligned}
& \left\langle\Delta \mid I^{(4)}\left(c_{0}\right)\right\rangle_{S}=\prod_{k=1}^{4} T^{(k)}\left(\beta^{(k)} ; N_{k}\right) \\
& =\left(c_{1}\right)^{-h_{1} / \hbar^{2}+b N Q}\left(\eta_{1}\right)^{-b\left(N_{2}+N_{3}+N_{4}\right)\left(b\left(N_{2}+N_{3}+N_{4}\right)+2 c_{0} / \hbar-Q\right)-\frac{b^{2}}{2}\left(N_{2}^{2}+N_{3}^{2}+N_{4}^{2}\right)+\frac{b Q}{2}\left(N_{2}+N_{3}+N_{4}\right)} \\
& \quad \times\left(\eta_{2}\right)^{-b\left(N_{3}+N_{4}\right)\left(b\left(N_{3}+N_{4}\right)+c_{0} / \hbar-Q\right)-b^{2}\left(N_{3}^{2}+N_{4}^{2}\right)+b Q\left(N_{3}+N_{4}\right)}\left(\eta_{3}\right)^{-b N_{4}\left(b N_{4}+c_{0} / \hbar-Q\right)-\frac{3}{2} b N_{4}\left(b N_{4}-Q\right)} \\
& \quad \times e^{-\frac{b c_{0}}{\hbar \eta_{1}}\left(\left(N_{2}-N_{3}\right)+\frac{2\left(N_{3}-N_{4}\right)}{\eta_{2}}-\frac{N_{3}-N_{4}}{3 \eta_{2}^{2}}+\frac{2 N_{4}}{\eta_{2} \eta_{3}}+\frac{N_{4}}{\eta_{2}^{2} \eta_{3}}-\frac{N_{4}}{\eta_{2}^{2} \eta_{3}^{2}}+\frac{N_{4}}{6 \eta_{2}^{2} \eta_{3}^{3}}\right)} .
\end{aligned}
$$




\section{Summary and discussion}

In this paper, the inner product of the irregular vector is studied which corresponds to the asymptotically free quivers of $\mathrm{SU}(2)$ gauge groups (general theories of $A_{1}$ class) [24-26]. The irregular vector is the simultaneous eigenstate of a set of positive Virasoro generators. The inner product of irregular vectors is obtained using the colliding limit of $(m+2)$ regular conformal block and is represented by the $\beta$-deformed Penner-type matrix model. The partition function becomes the two point correlation of irregular conformal block of rank $m$ and contains $(m+1)$-parameters. We have found explicitly the $m$-parametric dependence of the partition function.

As shown in section 2, we use the loop equation of the matrix model and find the parameter dependence of the inner product explicitly. In this process we need to evaluate the contour integral which is needed to eliminate the expectation values in terms of filling fraction and parameters. However, the contour integral in general gives elliptic function and the inverting process is very cumbersome to express the expectation values in terms of parameters including the filling fraction.

On the other hand, it is noted in section 3 that as far as the singular structure is concerned one may use a simple and powerful method. The method uses the flow equation of the partition function. The idea is based on the observation that the singular structure of the inner product is hierarchical. One finds that the singular part of the inner product between irregular vectors of rank $n$ and $m$ is factorized into those of inner product between regular and irregular vectors (3.7). Furthermore, the singular structure of the irregular vector rank $m$ can be factorized into those of lower ranks as shown in (3.16) and (3.17). Based on this hierarchical structure of the singularity, all the singular features are described by the effective partition function called $T^{(k)}(3.21)$

The advantage of using $T^{(k)}$ is that the self-consistency of the flow equations is enough to find all the singular structures of the partition function. We do not need the contour integration corresponding to the filling fraction. Why this method works lies in finding the initial condition for the flow equations. It is noted that two singular contributions, $H_{-1}^{(k)}$ and $H_{0}^{(k)}$ are responsible to $T^{(k)}$. The initial conditions needed for the singular part are trivially found from the analytical properties of the loop equation of the matrix model and are summarized in (3.42)-(3.46) which hold for all orders of large $N$ expansion. As the result, the singular part are determined exactly. This shows that there are $(m-1)$ types of instantons for the irregular state of rank $m$ and the corresponding filling fraction becomes the instanton number. The filling fraction is fixed during the colliding process but the instanton energy changes. Note that the instanton energy which is linear in the filling fraction in $H_{-1}^{(k)}$ and $H_{0}^{(k)}$ is found exactly to the all orders of the large $N$. The term quadratic in the filling fraction comes simply from the Vandermonde determinant. The chemical potential obtained this way is related with the $B$-cycle of the resolvent [27-29].

On the other hand, the behavior of the regular contribution $H_{n}^{(k)}$ for $n \geq 1$ is quite different. The initial condition is not found from the simple analytic structure of the loop equation. The initial condition needs to be found from other methods such as filling fraction integration. This is the reason why we can use the filling fraction integration in section 2 to find the regular contributions. 
Depending on the way of colliding limit, there may arise many-point irregular conformal block and more parameters appear. The irregular $n$-point conformal block has the Penner potential with singularity at $n$ points. One may see the same hierarchy of the singularity structure similar to 2-point irregular conformal block.

Note that $(m+1)$-parameters describe the irregular vector of rank $m$. Among them, $m$-parameter dependence is easily described in terms of the $m$-flow equations. However, the remaining one parameter dependence is not simple to find. As seen in the irregular vector with rank 1 , the remaining parameter dependence can be obtained from the colliding limit of the 3-point function of the regular conformal block. When $\beta=1$, the resulting partition function reduces to the original Penner model [9] and describes the pseudo Euler characteristics and is useful to understand $c=1$ string theory $[10,11]$. Therefore, the remaining parameter dependence should describe the generalized descendants of the irregular vector. The recent attempt to understand the certain limit of the regular conformal block in terms of Painlevé equation [30,31] will be useful to understand the remaining parameter dependence.

\section{Acknowledgments}

We thank A. Zamolodchikov and O. Lisovyy to draw attention to their recent works on Painlevé equation. This work is partially supported by the National Research Foundation of Korea (NRF) grant funded by the Korea government (MEST) 2005-0049409.

\section{A Self-consistency of the flow equation for $\tilde{T}^{(k)}$}

The flow equations of $\tilde{H}^{(k)}=-\hbar^{2} \log \tilde{T}^{(k)}$ is given in (3.29) and (3.30)

$$
\begin{aligned}
\frac{\partial \tilde{H}^{(k)}}{\partial \tau_{k}} & =-\frac{1}{\tau_{k}^{2}} \sum_{s=0}^{k-1} \sum_{j=k-s}^{k} \frac{\kappa_{j}}{j} A_{j-(k-s)} \tilde{D}_{s}, \\
\frac{\partial \tilde{H}^{(k)}}{\partial \kappa_{t}} & =\frac{1}{t \tau_{k}} \sum_{s=k-t}^{k-1} A_{s-(k-t)} \tilde{D}_{s}, \quad \text { for } 1 \leq t \leq k-2
\end{aligned}
$$

where $A_{0}=1$ and $A_{\ell}=-\left(A_{\ell-1}+\kappa_{k-2} A_{\ell-2}+\cdots+\kappa_{k-\ell} A_{0}\right)$. We use the special notations for some parameters $\tau_{k}=\kappa_{0}$ and $\kappa_{k-1}=\kappa_{k}=1$ and $\kappa_{\ell}=0$ for $\ell<0$. Therefore, $A_{\ell}=(-1)^{\ell}+\mathcal{O}\left(\left\{\kappa_{\ell}\right\}\right) . \tilde{D}_{s}$ is expanded in power series of $\tau_{k}, \kappa_{1}, \cdots, \kappa_{k-2}$;

$$
\tilde{D}_{s}=\sum\left[\tilde{D}_{s}\right]_{\alpha_{0}, \alpha_{1} \cdots, \alpha_{k-2}} \tau_{k}^{\alpha_{0}} \kappa_{1}^{\alpha_{1}} \cdots \kappa_{k-2}^{\alpha_{k-2}} .
$$

As demonstrated in section 3 up to $k=4$, the self-consistency of the flow equations (A.2) can fix all the singular parts of $\tilde{T}^{(k)}$. In this appendix, we prove that the self-consistency of the flow equations can determine all the singular parts of $\tilde{T}^{(k)}$ even for $k>4$. To prove this we use the self-consistency condition (3.27)

$$
\frac{\partial^{2} \tilde{H}^{(k)}}{\partial \kappa_{a} \partial \kappa_{b}}=\frac{\partial^{2} \tilde{H}^{(k)}}{\partial \kappa_{b} \partial \kappa_{a}} \quad(0 \leq a, b \leq k-2) .
$$


The flow equation shows that $\tilde{H}^{(k)}$ has the form (3.26)

$$
\tilde{H}^{(k)}\left(\left\{\kappa_{\ell}\right\}\right)=\frac{H_{-1}^{(k)}}{\tau_{k}}+H_{0}^{(k)} \log \tau_{k}+\sum_{n \geq 1} H_{n}^{(k)} \tau_{k}^{n} .
$$

This shows that the singular contribution to $T^{(k)}$ is due to the terms $H_{-1}^{(k)}$ and $H_{0}^{(k)}$. According to the consistency condition (A.4), $H_{0}^{(k)}$ is a constant independent of $\kappa_{\ell}$ 's whose value is found in (3.48). Therefore, we are going to concentrate on finding $H_{-1}^{(k)}$ which is completely fixed if one knows the $\tilde{D}_{s}$ at the limit $\tau_{k} \rightarrow 0$, i.e., $\left[\tilde{D}_{s}\right]_{\alpha_{0}, \alpha_{1} \cdots, \alpha_{k-2}}$ with $\alpha_{0}=0$.

To find $\tilde{D}_{s}$ from the consistency condition (A.4) one needs some elementary information on $\tilde{D}_{s}$. This is obtained from the loop equation. The loop equation has the form (3.34)

$$
4 \tilde{W}(z)^{2}-4 \tilde{V}^{\prime}(z) \tilde{W}(z)+2 \hbar Q \tilde{W}^{\prime}(z)-\hbar^{2} \tilde{W}(z, z)=\tilde{f}(z)=\frac{1}{\tau_{k}} \sum_{s=0}^{k-1} \frac{\tilde{D}_{s}}{z^{2+s}} .
$$

Large $z$ expansion provides useful results on $\tilde{D}_{s}$ as shown in (3.41). Some information we need are listed as follows:

$$
\begin{aligned}
{\left[\tilde{D}_{k-1}\right]_{\alpha_{0}=0, \alpha_{1}=0, \cdots, \alpha_{k-2}=0} } & =2 \hbar b N_{k} \beta^{(k)} \\
{\left[\tilde{D}_{s}\right]_{0, \cdots, 0, \alpha_{t}=1,0, \cdots, 0} } & =(-1)^{s-t} 2 \hbar b N_{k} \beta^{(k)} \quad(1 \leq t \leq s \leq k-2)
\end{aligned}
$$

and for $s \leq k-2$

$$
\left[\tilde{D}_{s}\right]_{0, \cdots, 0, \alpha_{s}, \alpha_{s+1}, \cdots, \alpha_{k-2}} \equiv 0 \quad \text { unless } \quad \alpha_{s}=1, \quad \alpha_{s+1}=\cdots=\alpha_{k-2}=0 .
$$

Note that the information holds to the all order of large $N$. We present how to find $\tilde{D}_{s}$ in the following steps.

Step [1]: Find $\tilde{D}_{\boldsymbol{k}-1}$ in power series in $\boldsymbol{\kappa}_{\boldsymbol{k}-2}$. Use the consistency flow along $\tau_{k}$ and $\kappa_{k-2}$

$$
\frac{\partial^{2} \tilde{H}^{(k)}}{\partial \kappa_{k-2} \partial \tau_{k}}=\frac{\partial^{2} \tilde{H}^{(k)}}{\partial \tau_{k} \partial \kappa_{k-2}}
$$

and find $\tilde{D}_{k-1}$ in power series in $\kappa_{k-2}$. This will determine $\left[\tilde{D}_{k-1}\right]_{\alpha_{0}=0, \alpha_{1}=0, \cdots, \alpha_{k-3}=0, \alpha_{k-2}}$ with $\alpha_{k-2} \geq 0$.

The consistency condition gives

$$
\sum_{s=0}^{k-1} \sum_{j=k-s}^{k} \frac{\kappa_{j}}{j} \frac{\partial}{\partial \kappa_{k-2}}\left(A_{j-(k-s)} \tilde{D}_{s}\right)=-\frac{\tau_{k}}{k-2} \frac{\partial}{\partial \tau_{k}}\left(\sum_{s=k-t}^{k-1} A_{s-2} \tilde{D}_{s}\right)
$$

When $\tau_{k} \rightarrow 0$, r.h.s. of (A.11) obviously vanishes. In addition, the initial condition (A.9) shows that $\tilde{D}_{s}=0$ for $0 \leq s \leq k-3$ when $\left(\tau_{k}, \kappa_{1}, \cdots, \kappa_{k-3}\right) \rightarrow 0$, and therefore, the equation (A.11) simplifies to

$$
\left(\sum_{j=k-2}^{k} \frac{\kappa_{j} A_{j-1}}{j}\right) \tilde{D}_{k-1}^{\prime}+\left(\sum_{j=k-2}^{k} \frac{\kappa_{j} A_{j-1}^{\prime}}{j}\right) \tilde{D}_{k-1}=-\sum_{j=k-2}^{k} \frac{\kappa_{j}}{j}\left(A_{j-2} \tilde{D}_{k-2}\right)^{\prime}
$$


where $X^{\prime}$ denotes derivative with respect to $\kappa_{k-2}$. The initial condition (A.8) shows that $\tilde{D}_{k-2}=2 \hbar b N_{k} \beta^{(k)} \kappa_{k-2}$. Therefore, (A.12) is the inhomogeneous first oder equation of $\tilde{D}_{k-1}$, which gives the simple recursion relation for the power series in $\kappa_{k-2}$. This fixes $\tilde{D}_{k-1}$ in power series in $\kappa_{k-2}$ with the initial condition $\left[\tilde{D}_{k-1}\right]_{\kappa_{k-2}=0}=2 \hbar b N_{k} \beta^{(k)}$ as given in (A.7).

Step [2]: Find $\tilde{D}_{k-2}$ and $\tilde{D}_{k-1}$ in power series in $\kappa_{k-3}$ and $\kappa_{k-2}$. Next step is to use the consistency flow along $\tau_{k}, \kappa_{k-3}$ and $\kappa_{k-2}$.

$$
\frac{\partial^{2} \tilde{H}^{(k)}}{\partial \tau_{k} \partial \kappa_{k-3}}=\frac{\partial^{2} \tilde{H}^{(k)}}{\partial \kappa_{k-3} \partial \tau_{k}}, \quad \frac{\partial^{2} \tilde{H}^{(k)}}{\partial \kappa_{k-2} \partial \kappa_{k-3}}=\frac{\partial^{2} \tilde{H}^{(k)}}{\partial \kappa_{k-3} \partial \kappa_{k-2}} .
$$

This additional two equations provide the recursion relations for $\tilde{D}_{k-1}$ and $\tilde{D}_{k-2}$ in power series of $\kappa_{k-3}$ and $\kappa_{k-2}$ when $\left\{\tau_{k}, \kappa_{1}, \cdots, \kappa_{k-4}\right\} \rightarrow 0$. Using the result obtained from the step [1], the coefficients $\left[\tilde{D}_{k-1}\right]_{\alpha_{0}=0, \alpha_{1}=0, \cdots, \alpha_{k-4}=0, \alpha_{k-3}>0, \alpha_{k-2} \geq 0}$ and $\left[\tilde{D}_{k-2}\right]_{\alpha_{0}=0, \alpha_{1}=0, \cdots, \alpha_{k-4}=0, \alpha_{k-3}>0, \alpha_{k-2} \geq 0}$ are determined.

Step [3]: Inductive proof of finding $\tilde{D}_{\ell}$ 's in power series in $\kappa_{t}, \cdots \kappa_{k-1}$. Suppose the coefficients of $\tilde{D}_{\ell}$

$$
\left[\tilde{D}_{\ell}\right]_{0, \cdots, 0, \alpha_{t+1}, \alpha_{t+2}, \cdots, \alpha_{k-2}} \text { for }(t+2 \leq \ell \leq k-1)
$$

is known for $\left(\alpha_{t+1}, \cdots, \alpha_{k-2}\right) \geq 0$. The case $t=(k-3)$ is done in the step [1], which is true. Therefore, our proof can be done using the inductive way.

Suppose the coefficient in (A.14) is known for a certain $t<k-3$. Our claim is that the consistency conditions

$$
\frac{\partial^{2} \tilde{H}^{(k)}}{\partial \kappa_{t} \partial \tau_{k}}=\frac{\partial^{2} \tilde{H}^{(k)}}{\partial \tau_{k} \partial \kappa_{t}}, \quad \frac{\partial^{2} \tilde{H}^{(k)}}{\partial \kappa_{t} \partial \kappa_{a}}=\frac{\partial^{2} \tilde{H}^{(k)}}{\partial \kappa_{a} \partial \kappa_{t}} \text { for }(t+1 \leq a \leq k-2),
$$

will fix the next coefficient $\left[\tilde{D}_{\ell}\right]_{0, \cdots, 0, \alpha_{t}, \alpha_{t+1}, \cdots, \alpha_{k-2}}$ for $(t+1 \leq \ell \leq k-1)$.

To prove this claim, let us use the consistency conditions (A.15) to get

$$
\begin{aligned}
\sum_{s=0}^{k-1} \sum_{j=k-s}^{k} \frac{\kappa_{j}}{j} \frac{\partial}{\partial \kappa_{t}}\left(A_{j-(k-s)} \tilde{D}_{s}\right) & =-\frac{\tau_{k}}{t} \frac{\partial}{\partial \tau_{k}}\left(\sum_{s=k-t}^{k-1} A_{s-(k-t)} \tilde{D}_{s}\right) \\
\frac{\partial}{\partial \kappa_{t}}\left(\sum_{s=k-a}^{k-1} A_{s-(k-a)} \tilde{D}_{s}\right) & =\frac{a}{t} \frac{\partial}{\partial \kappa_{a}}\left(\sum_{s=k-t}^{k-1} A_{s-(k-t)} \tilde{D}_{s}\right) .
\end{aligned}
$$

We further reduce the above equations using the known information. Note that we are trying to find the solution at $\tau_{k}=0$ and $\left\{\kappa_{1}, \cdots, \kappa_{t-1}\right\}=0$. Therefore, we put $\tau_{k} \rightarrow 0$ and discard r.h.s. of (A.16). In addition, $\tilde{D}_{s}=0$ for $s=0, \cdots, t-1$ by the conditions (A.8) and (A.9). Therefore, the non-vanishing components in the above equations are simplified.

$$
\begin{aligned}
\sum_{s=t}^{k-1} \sum_{j=k-s \geq t}^{k} \frac{\kappa_{j}}{j} \frac{\partial}{\partial \kappa_{t}}\left(A_{j-(k-s)} \tilde{D}_{s}\right) & =0 \\
\frac{\partial}{\partial \kappa_{t}}\left(\sum_{s=t}^{k-1} A_{s-(k-a)} \tilde{D}_{s}\right) & =\frac{a}{t} \frac{\partial}{\partial \kappa_{a}}\left(\sum_{s=t}^{k-1} A_{s-(k-t)} \tilde{D}_{s}\right) .
\end{aligned}
$$


Note that we put the lower limit of the summation $s=t$ in (A.19) using $A_{\ell}=0$ when $\ell<0$, without which the lower limit should be $s=\max (k-a, t)$ (l.h.s.) and $s=\max (k-t, t)$ (r.h.s.).

Note that $\tilde{D}_{t}$ is known and linear in $\kappa_{t}$ in (A.8) and (A.9). The unknowns are $\tilde{D}_{s}$ with $(t+1) \leq s \leq(k-1)$. Therefore, it is convenient to put the equations (A.18) and (A.19) in a simple product form

$$
\sum_{s=t+1}^{k-1} \tilde{A}_{s} \tilde{D}_{s}^{\prime}=B_{t}, \quad \sum_{s=t+1}^{k-1} A_{s-(k-a)} \tilde{D}_{s}^{\prime}=B_{a} \text { for }(t+1 \leq a \leq k-2)
$$

where $X^{\prime}$ denotes derivative with respect to $\kappa_{t}$. $\tilde{A}_{s}$ is the weighted sum of $A_{s}, \tilde{A}_{s}=$ $\sum_{j=k-s}^{k}\left(\kappa_{j} A_{j-(k-s)} / j\right)$ and $B_{t}$ and $B_{a}$ contain $\tilde{D}_{s}$ 's but no derivatives of $\kappa_{t}$ 's.

$$
\begin{aligned}
B_{t} & =-\sum_{s=t}^{k-1} \sum_{j=k-s}^{k} \frac{\kappa_{j} A_{j-(k-s)}^{\prime} \tilde{D}_{s}}{j}-\tilde{A}_{t} \tilde{D}_{t}^{\prime} \\
B_{a} & =\frac{a}{t} \frac{\partial}{\partial \kappa_{a}}\left(\sum_{s=k-t}^{k-1} A_{s-(k-t)} \tilde{D}_{s}\right)-\delta_{t, k-a} \tilde{D}_{t}^{\prime} .
\end{aligned}
$$

where $\tilde{D}_{t}^{\prime}=2 \hbar N_{k} \beta^{(k)}$.

Putting the new equations in a matrix form $\mathbb{A D}^{\prime}=\mathbb{B}$, we have $(k-t-1) \times(k-t-1)$ invertible matrix $\mathbb{A}$

$$
\mathbb{A}:=\left(\begin{array}{cccc}
\tilde{A}_{t+1} & \tilde{A}_{t+2} & \cdots \cdots & \tilde{A}_{k-1} \\
A_{t-1} & A_{t} & \cdots \cdots & A_{k-3} \\
A_{t-2} & A_{t-1} & \cdots \cdots & A_{k-4} \\
\vdots & \vdots & & \vdots \\
\vdots & \vdots & & \vdots \\
A_{t-(k-t-2)} & A_{t-(k-t-3)} & \cdots & A_{t}
\end{array}\right)
$$

and $k-t-1$ column vectors $\mathbb{D}$ and $\mathbb{B}$

$$
\mathbb{D}=\left(\begin{array}{c}
\tilde{D}_{t+1} \\
\vdots \\
\tilde{D}_{k-1}
\end{array}\right), \quad \mathbb{B}=\left(\begin{array}{c}
B_{t} \\
\vdots \\
B_{k-2}
\end{array}\right)
$$

Inverting the matrix equation we have $\mathbb{D}^{\prime}=\mathbb{A}^{-1} \mathbb{B}$. When $\mathbb{D}$ is put in power series of $\kappa_{t}, \mathbb{D}=\sum_{a \geq 0} \mathbb{D}_{a} \kappa_{t}^{a}$, the inverted equation provides the recursion relation of the $\mathbb{D}_{a}$ 's. which is solved if the initial condition $\mathbb{D}_{0}$ is known. Note that $\mathbb{D}_{0}$ is the assumption of our claim (A.14). Therefore, the claim is proved.

Note that the iteration goes from $t=(k-3)$ to $t=1$. The procedure determines all the contribution to $\tilde{D}_{\ell}(2 \leq \ell \leq k-1)$ when $\tau_{k}=0$. The remaining $\tilde{D}_{1}$ is already known completely in (A.8) and (A.9) when $\tau_{k}=0$. 
Open Access. This article is distributed under the terms of the Creative Commons Attribution License (CC-BY 4.0), which permits any use, distribution and reproduction in any medium, provided the original author(s) and source are credited.

\section{References}

[1] P. Francesco, P. Mathieu and D. Senechal, Conformal Field Theory, Springer-Verlag, New York (1997).

[2] D. Gaiotto, Asymptotically free $N=2$ theories and irregular conformal blocks, arXiv:0908.0307 [INSPIRE].

[3] A. Marshakov, A. Minorov and A. Morozov, On non-conformal limit of the AGT relations, Phys. Lett. B 682 (2009) 125 [arXiv:0909. 2052] [INSPIRE].

[4] E. Felinska, Z. Jaskolski and M. Kosztolowicz, Whittaker pairs for the Virasoro algebra and the Gaiotto - BMT states, J. Math. Phys. 53 (2012) 033504 [Erratum ibid. 53 (2012) 129902] [arXiv: 1112.4453] [INSPIRE].

[5] G. Bonelli, K. Maruyoshi and A. Tanzini, Wild Quiver Gauge Theories, JHEP 02 (2012) 031 [arXiv:1112.1691] [INSPIRE].

[6] D. Gaiotto and J. Teschner, Irregular singularities in Liouville theory and Argyres-Douglas type gauge theories, I, JHEP 12 (2012) 050 [arXiv:1203.1052] [INSPIRE].

[7] P. Argyres and M. Douglas, New phenomena in SU(3) supersymmetric gauge theory, Nucl. Phys. B 448 (1995) 93 [hep-th/9505062] [INSPIRE].

[8] P.C. Argyres, M.R. Plesser, N. Seiberg and E. Witten, New $N=2$ superconformal field theories in four-dimensions, Nucl. Phys. B 461 (1996) 71 [hep-th/9511154] [INSPIRE].

[9] R.C. Penner, Perturbative series and the moduli space of Riemann surface, J. Diff. Geo. 27 (1998) 35 .

[10] J. Distler and C. Vafa, A Critical matrix model at $c=1$, Mod. Phys. Lett. A 6 (1991) 259 [INSPIRE].

[11] S. Chaudhuri, H. Dykstra and J.D. Lykken, The Penner matrix model and $C=1$ strings, Mod. Phys. Lett. A 6 (1991) 1665 [INSPIRE].

[12] R. Dijkgraaf and C. Vafa, Toda Theories, Matrix Models, Topological Strings and $N=2$ Gauge Systems, arXiv:0909.2453 [INSPIRE].

[13] L.F. Alday, D. Gaiotto and Y. Tachikawa, Liouville Correlation Functions from Four-dimensional Gauge Theories, Lett. Math. Phys. 91 (2010) 167 [arXiv:0906.3219] [INSPIRE].

[14] T. Eguchi and K. Maruyoshi, Penner Type Matrix Model and Seiberg-Witten Theory, JHEP 02 (2010) 022 [arXiv:0911.4797] [INSPIRE].

[15] T. Nishinaka and C. Rim, $\beta$-Deformed Matrix Model and Nekrasov Partition Function, JHEP 02 (2012) 114 [arXiv:1112.3545] [INSPIRE].

[16] T. Nishinaka and C. Rim, Matrix models for irregular conformal blocks and Argyres-Douglas theories, JHEP 10 (2012) 138 [arXiv:1207.4480] [INSPIRE].

[17] E. Brézin, C. Itzykson, G. Parisi and J.B. Zuber, Planar Diagrams, Commun. Math. Phys. 59 (1978) 35 [inSPIRE]. 
[18] J. Ambjørn, L. Chekhov, C.F. Kristjansen and Y. Makeenko, Matrix model calculations beyond the spherical limit, Nucl. Phys. B 404 (1993) 127 [Erratum ibid. B 449 (1995) 681] [hep-th/9302014] [INSPIRE].

[19] G. Akemann, Higher genus correlators for the Hermitian matrix model with multiple cuts, Nucl. Phys. B 482 (1996) 403 [hep-th/9606004] [INSPIRE].

[20] A.B. Zamolodchikov and A.B. Zamolodchikov, Structure constants and conformal bootstrap in Liouville field theory, Nucl. Phys. B 477 (1996) 577 [hep-th/9506136] [INSPIRE].

[21] H. Dorn and H.J. Otto, Two and three point functions in Liouville theory, Nucl. Phys. B 429 (1994) 375 [hep-th/9403141] [INSPIRE].

[22] M. Hanada et al., Loops versus matrices: The Nonperturbative aspects of noncritical string, Prog. Theor. Phys. 112 (2004) 131 [hep-th/0405076] [INSPIRE].

[23] M. Mariño, R. Schiappa and M. Weiss, Multi-Instantons and Multi-Cuts, J. Math. Phys. 50 (2009) 052301 [arXiv:0809.2619] [INSPIRE].

[24] E. Witten, Solutions of four-dimensional field theories via M-theory, Nucl. Phys. B 500 (1997) 3 [hep-th/9703166] [INSPIRE].

[25] D. Gaiotto, N=2 dualities, JHEP 08 (2012) 034 [arXiv:0904.2715] [INSPIRE].

[26] D. Gaiotto, G.W. Moore and A. Neitzke, Wall-crossing, Hitchin Systems and the WKB Approximation, arXiv:0907.3987 [INSPIRE].

[27] L. Chekhov and B. Eynard, Hermitean matrix model free energy: Feynman graph technique for all genera, JHEP 03 (2006) 014 [hep-th/0504116] [INSPIRE].

[28] A. Mironov, A. Morozov and S. Shakirov, Matrix Model Conjecture for Exact BS Periods and Nekrasov Functions, JHEP 02 (2010) 030 [arXiv:0911.5721] [INSPIRE].

[29] A. Mironov, A. Morozov and A. Morozov, Conformal blocks and generalized Selberg integrals, Nucl. Phys. B 843 (2011) 534 [arXiv:1003.5752] [INSPIRE].

[30] O. Gamayun, N. Iorgov and O. Lisovyy, How instanton combinatorics solves Painlevè VI, V and IIIs, J. Phys. A 46 (2013) 335203 [arXiv:1302.1832] [INSPIRE].

[31] A. Litvinov, S. Lukyanov, N. Nekrasov and A. Zamolodchikov, Classical Conformal Blocks and Painleve VI, arXiv:1309.4700 [INSPIRE]. 